\title{
The role of atmosphere and ocean physical processes in ENSO in a perturbed physics coupled climate model
}

\author{
S. Y. Philip ${ }^{1}$, M. Collins ${ }^{2}$, G. J. van Oldenborgh ${ }^{1}$, and B. J. J. M. van den Hurk ${ }^{1,3}$ \\ ${ }^{1}$ Royal Netherlands Institute of Meteorology, De Bilt, The Netherlands \\ ${ }^{2}$ Met Office Hadley Centre, Exeter, UK \\ ${ }^{3}$ Institute for Marine and Atmospheric Research, Utrecht University, Utrecht, The Netherlands
}

Received: 27 July 2009 - Published in Ocean Sci. Discuss.: 9 September 2009

Revised: 26 March 2010 - Accepted: 8 April 2010 - Published: 20 April 2010

\begin{abstract}
We examine the behaviour of the El Niño - Southern Oscillation (ENSO) in an ensemble of global climate model simulations with perturbations to parameters in the atmosphere and ocean components respectively. The influence of the uncertainty in these parametrisations on ENSO are investigated systematically. The ensemble exhibits a range of different ENSO behaviour in terms of the amplitude and spatial structure of the sea surface temperature (SST) variability. The nature of the individual feedbacks that operate within the ENSO system are diagnosed using an Intermediate Complexity Model (ICM), which has been used previously to examine the diverse ENSO behaviour of the CMIP3 multi-model ensemble. Unlike in that case, the ENSO in these perturbed physics experiments is not principally controlled by variations in the mean climate state. Rather the parameter perturbations influence the ENSO characteristics by modifying the coupling feedbacks within the cycle. The associated feedbacks that contribute most to the ensemble variations are the response of SST to local wind variability and damping, followed by the response of SST to thermocline anomalies and the response of the zonal wind stress to those SST anomalies. Atmospheric noise amplitudes and oceanic processes play a relatively minor role.
\end{abstract}

Correspondence to: $\mathrm{M}$. Collins (matthew.collins@metoffice.gov.uk)

\section{Introduction}

Coupled numerical models (GCMs) now form the core of efforts to predict natural climate variability and forced climate change on time scales of seasons, decades and centuries. They also form the basis of a large number of studies which seek to understand the mechanisms for those variations in climate. The El Niño - Southern Oscillation (ENSO) presents a considerable challenge for numerical models as the different physical (and biogeochemical) processes that need to act together to produce an oscillation are diverse; ranging from large to small-scale oceanic dynamics, atmospheric dynamics, cloud processes, surface fluxes etc.

There have been some notable advances in recent years in the ability of models to simulate ENSO. AchutaRao and Sperber (2006) track the ENSO-ability of models during two development cycles and note that the majority of the models in the most recent collection now has the ability to spontaneously produce an oscillation that has characteristics that resemble those that are observed in the real-world ENSO. Numerous studies (e.g. van Oldenborgh et al., 2005; Guilyardi, 2006) however note that there is still a wide range of model ability in terms of the basic characteristics of amplitude, period, phase locking to the annual cycle etc. Recent efforts have sought understanding of those basic characteristics in terms of the physical feedbacks that are involved in ENSO (e.g. Philip and van Oldenborgh, 2006; Dewitte et al., 2007; Philip and van Oldenborgh, 2009). Such diagnostics and metrics (Guilyardi et al., 2009) are currently being employed in efforts to reduce model "errors" with a view to correcting and improving models or in assigning relative skill of different models in probabilistic projections. 
It is useful to separate model errors that affect the ability to simulate ENSO into two types. The first type includes errors or biases in the mean climate state; both ocean and atmosphere errors as well as errors that are in some way coupled, including errors in the seasonal cycle, are ubiquitous. Typical biases include the simulation of a cold tongue that is too cold and too extensive and the simulation of a South Pacific Convergence Zone that is too zonally oriented (Lin, 2007): the so-called "double-ITCZ" problem. Other errors have also been described (e.g. Guilyardi, 2006). Mean-state errors develop quickly during model simulations and hence are often subject to much directed effort to reduce them.

The second type of potential model error is associated with inaccuracies in the physical processes involved in ENSO. For example, errors in the strength of the Bjerknes feedback whereby anomalies in sea surface temperatures (SSTs) force variations in the atmospheric winds and circulation that tend to reinforce those SST anomalies (a positive feedback). Such feedback processes are increasingly the focus of GCM ENSO studies in the literature (e.g. Philip and van Oldenborgh, 2006, 2009; Lloyd et al., 2009; Guilyardi et al., 2009).

The difficulty in separating model errors in this way is that they are clearly linked. Mean-state errors, for example, produce errors in the mean distribution of clouds, which may then affect the pattern and strength of surface-flux damping of ENSO SST anomalies. Likewise, errors in the surfaceflux feedback may lead, non-linearly, to errors in mean-state SSTs. Our ability to understand ENSO errors in models and ultimately improve the baseline simulation of ENSO is complicated by such interactions (Philip and van Oldenborgh, 2010; Guilyardi et al., 2009).

Here we partly circumvent this problem by examining the simulation of ENSO in a set of model experiments with perturbations to key atmospheric and oceanic parameters. In these so-called "perturbed physics" experiments, the mean climate state and annual cycle are, to a large extend, controlled by imposing flux adjustment terms, which tie the model SSTs and salinities close to observed values. While the elimination of flux adjustment terms has been seen as a breakthrough in climate modelling (e.g. Gordon et al., 2000), non-flux-adjusted models suffer from biases in the mean state and seasonal cycle (Guilyardi, 2006).

In this case the flux adjustments serve to minimise the mean-state errors and allow us to examine the physical processes involved in ENSO in some detail. As coupled models are being improved, it is expected that mean-state errors will continue to reduce, which eventually enables the improvement of the realism of physical feedbacks in models in a more straightforward way (i.e. without the complication of errors in the mean state). This study anticipates such a situation.

We adopt the same approach as used in Philip and van Oldenborgh (2009) in which an "Intermediate Complexity Model" (ICM) is fitted to different GCMs to examine the role of both linear feedback loops and the non-linear role of atmospheric noise. The ICM can qualitatively reproduce the basic characteristics of the ENSO behaviour in the individual CMIP3 GCMs when the parameters of the model are fitted to the GCM output. The different feedbacks are shown in Fig. 1 and described in more detail in Sect. 3.

In a diverse multi-model ensemble, it is difficult to investigate the influence of each part of the ENSO feedback loop separately, as all components differ from each other. The fitted ICM is numerically stable as long as both the atmosphere and the ocean are fitted to the same GCM. The ICM is not necessarily numerically stable when using atmosphere parameters of one GCM and ocean parameters of another GCM. The variations in ENSO feedbacks are too large to put parts of the feedback loop from different models together. This problem is much less acute in the case of the perturbed physics ensemble examined here. Coupling of the ocean of one ensemble member with the atmosphere of another member often gives more consistent ICM runs than performing the same exercise with the parameters fitted from two very different GCMs. This proves to be a useful tool in understanding the behaviour of the different perturbed physics GCMs.

The approach is complementary to Toniazzo et al. (2008) who test the variation of ENSO characteristics in a very similar model ensemble. In those experiments, the same HadCM3 atmospheric parameters are perturbed, but with slightly different values. Here we use combinations based on a subset of a much larger ensemble described in Webb et al. (2006) in which a simple slab ocean is used instead of a fully dynamical ocean. In addition, the flux adjustment terms are calculated in a slightly different manner to reduce N. Atlantic and Arctic SST and sea-ice biases and, in this study, we also perturb parameters in the ocean component of the model.

In the Toniazzo et al. (2008) study, ensemble members are grouped into subsets of the ensemble with low and high ENSO variability respectively. They find a prevalence for the mode of ENSO which principally involves interactions with the atmosphere and upper ocean (the so-called SST mode e.g. Fedorov and Philander (2001) and trace the variations in the amplitude of ENSO in the different members to variations in the low-cloud cover in the east Pacific and to non-ENSOrelated variability in the south-east tropical Pacific. They only find a weak negative relation between ENSO strength and wind response to SST.

Here we quantify the influence of different coupling and atmospheric noise parameters of ENSO separately. We examine an updated version of the perturbed parameter ensemble used by Toniazzo et al. (2008). The influence of the different parts of the ENSO feedback loop is tested in the context of the ICM. This enables us to choose parts of the ENSO feedback loop, individually fit them to different model runs and test the impact relative to a reference run. In the reference ICM we mutually exchange fit parameters from different perturbed physics GCM ensemble members. In this way the influence of each parameter can be quantified separately. 
The objective is to quantify the importance of different parts in the linear ENSO feedback loop on variations in ENSO.

The HadCM3 atmospheric parameter perturbed ensemble is presented in Sect. 2. The different parts of the feedback loop are described in Sect. 3. In Sect. 4 ENSO characteristics of the HadCM3 atmospheric parameter perturbed ensemble (see Sect. 2) are briefly discussed. The terms of the conceptual model are fitted to the data in Sect. 5. The relations between these terms and ENSO characteristics are investigated in Sect. 6. Section 7 presents conclusions.

\section{Perturbed physics GCM experiments}

The "perturbed physics approach" was developed in response to the call for better quantification of uncertainties in climate projections (see Chapter 14 of the IPCC Third Assessment Report, e.g. Moore et al., 2001). The basic approach involves a single model structure in which perturbations are applied to the values of a range of presumably uncertain parameters; the determination of the range of the parameters is based on discussions with colleagues involved in parameterisation development and/or surveys of the climate modelling literature. In some cases, different variants of physical schemes may be also be switched on or off. Also parameters in those alternative schemes are varied. Any experiment that is routinely performed with single models can be produced in "ensemble mode" subject to constraints on computer time. A significant amount of perturbed physics experimentation has been done with HadCM3 and variants, starting with the work of Murphy et al. (2004) and Stainforth et al. (2005) and continuing with, for example, Piani et al. (2005); Webb et al. (2006); Knutti et al. (2006); Collins et al. (2006); Harris et al. (2006); Collins (2007); Sanderson and Piani (2007); Sanderson et al. (2008); Rougier et al. (2009). Nevertheless, other modelling centres are also investigating the approach using GCMs (e.g. Annan et al., 2005; Niehörster et al., 2006) and more simplified models (e.g. Schneider von Deimling et al., 2006) with a view to both understanding the behaviour of their models and to quantifying uncertainties in predictions.

Here we make use of perturbed physics ensembles produced with the version of HadCM3 in which a fully dynamical ocean and atmosphere are coupled. HadCM3 has the advantage that the model is structurally capable of simulating key aspects of ENSO as has been noted in a number of studies (Collins et al., 2001; AchutaRao and Sperber, 2006, 2002; van Oldenborgh et al., 2005; Toniazzo, 2006; Guilyardi, 2006). While small-scale processes which affect ENSO are clearly not captured by such a relatively low resolution model, the main large-scale physical feedbacks and processes can be. In our experiments, two ensembles are used of 16 members each. In one ensemble (hereafter ATMensemble, members 1-16), perturbations are only applied to parameters in the atmosphere component of the model, the ocean parameters being held fixed at their standard settings.

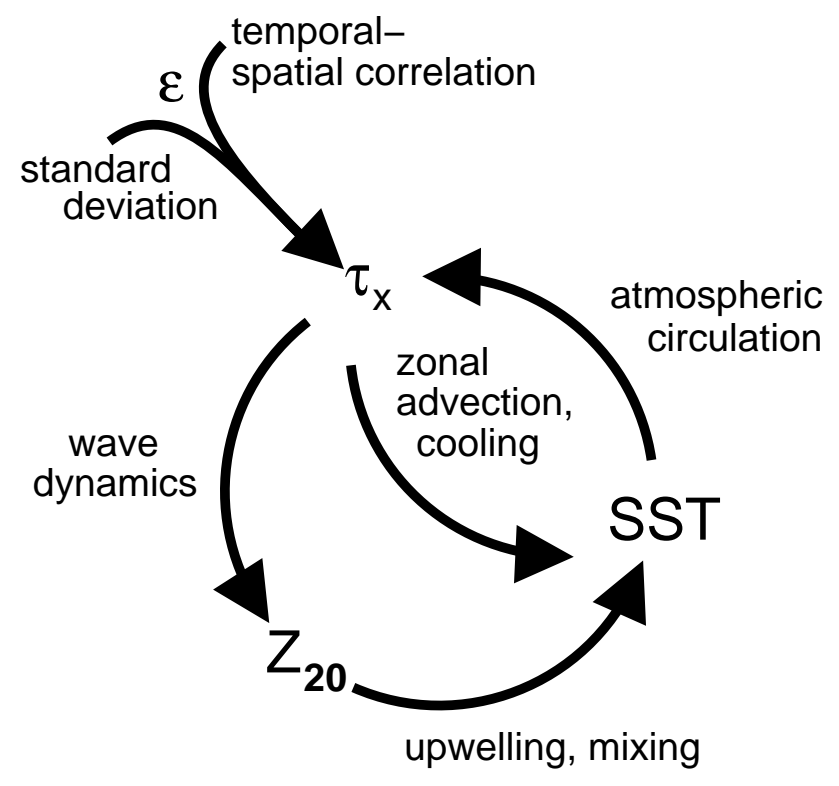

Fig. 1. The main feedbacks between wind stress $\left(\tau_{x}\right)$, SST and thermocline depth $\left(Z_{20}\right)$ in the ENSO phenomenon and the external noise term $\epsilon$.

In the second (hereafter OCN-ensemble, members 17-32), perturbations are only applied to parameters in the ocean component of the model, the atmosphere parameters being held fixed at their standard settings. The run with standard model parameter settings is denoted STAM. STAM, ATM and OCN thus comprise a total of 33 members. The 16 sets of atmosphere-parameter settings are chosen in order to sample a range of atmosphere feedbacks relevant to climate change, to span a range of parameter values and to maximise the chance of getting model versions that have time-mean climates that are as close as possible to observations for a number of observed climate fields. The algorithm for choosing the ATM-ensemble parameters is described in Webb et al. (2006). In the case of perturbations to the ocean parameters (OCN-ensemble) a slightly different approach is taken. For this ensemble, Latin-hypercube sampling of parameters that control horizontal mixing of heat and momentum, the vertical diffusivity of heat, isopycnal mixing, mixed layer processes and water type is performed. Despite this difference in sampling strategy, it will be demonstrated that both atmospheric and oceanic ENSO-processes are sufficiently perturbed to produce a wide range of different ENSO behaviour that can be diagnosed using the ICM approach. ? discusses the experimental setup and aspects of global-model evaluation and feedbacks in some detail.

It should be noted that the experiments used here are an updated version of those used in Collins et al. (2006) and Toniazzo et al. (2008) in which also ENSO characteristics are examined. In those ensemble experiments, significant SST and sea-ice biases arise in the North Atlantic and Artic 
oceans because of the particular implementation of fluxadjustments during the spin-up phase. Monthly-mean flux adjustments were employed to (i) prevent model drift that would result from perturbations to the parameters that lead to top-of-atmosphere net flux imbalances, and (ii) to improve the credibility of the simulations in simulating regional climate change and feedbacks. The spin-up technique used in the experiments examined here is similar to that described in Collins et al. (2006) except that a less vigorous salinity relaxation is employed during the Haney-forced phase, in which SSTs and surface salinities are relaxed toward a seasonallyvarying climatology - see Collins et al. (2006). This is found to significantly alleviate the problem of SST and sea-ice biases found in the Collins et al. (2006) ensemble. It is unlikely that errors in simulated North Atlantic and Arctic climate would affect ENSO variability directly (although some authors have highlighted the possibility of remote interactions e.g. Timmermann et al. (2007), hence comparisons with the findings of the Toniazzo et al. (2008) study are possible.

It should be noted that the flux-adjustment terms apply the same annually varying fluxes of heat and freshwater throughout the experiments. Hence they are invariant with respect to interannual variations in SST, winds and other dynamical variables. Other components of the surface heat flux balance; sensible and latent heating, short wave (SW) and long wave (LW) fluxes, can and do vary considerably in concert with variations in SST, winds, clouds etc. While it has been show that flux-adjustments can influence ENSO coupling processes in reduced complexity models (Dijkstra and Neelin , 1995) their role in impacting the ENSO cycle in fully dynamical coupled models is less well understood. Flux adjustments are essential here to prevent considerable model drift that would result in baseline climates much removed from reality and consequently of little interest. In a related project, some perturbed physics versions of HadCM3 have been defined in which the net radiative balance is close to zero and hence flux adjustments are not needed. In these preliminary experiments, the ENSO shows a similar range of behaviour to that presented here suggesting that the fluxadjustments do not limit the validity of this study. Nevertheless, further research should be performed to address this issue as flux-adjusted perturbed physics ensembles have become central in efforts to quantify uncertainty and provide probabilistic climate prediction (Collins, 2007).

\section{Method: the Intermediate Complexity Model}

The separate contributions of the main components that contribute to the characteristics of ENSO are shown schematically in Fig. 1 (see also van Oldenborgh et al., 2005; Philip and van Oldenborgh, 2010). In this conceptual model of ENSO, the main interactions are separated. These are the influence of wind stress on thermocline depth, the impact of SST anomalies on wind stress and the dependence of SST on both thermocline depth and on wind stress. External atmospheric noise also influences ENSO.

The terms shown in Fig. 1 are represented in the ICM using statistical relationships derived from either observations or GCM output. They can be changed independently from each other or in combination in order to study the influence of the different components separately on ENSO. In the next subsections we describe the ICM in more detail and elaborate on the couplings and noise terms.

\subsection{Basic structure and experiments with the ICM}

The equatorial Pacific ICM is based on the so-called Gmodel (Burgers et al., 2002; Burgers and van Oldenborgh, 2003). The Gmodel consists of a linear 1.5-layer shallow-water ocean model with a gravest baroclinic mode, a linear statistical atmosphere and a linear SST anomaly equation. The ICM version used in this study is additionally driven by physically consistent atmospheric noise patterns such that the characteristics of the noise that are most important for ENSO are captured (Philip and van Oldenborgh, 2009).

The model domain ranges from $30^{\circ} \mathrm{S}$ to $30^{\circ} \mathrm{N}$ and $122^{\circ} \mathrm{E}$ to $72^{\circ} \mathrm{W}$, on a $2^{\circ} \times 1^{\circ}$ longitude-latitude grid with realistic coast lines. The ocean model solves the shallow water equations (Gill, 1982) with an integration time-step of 1/3 day. In this study the length of each ICM run is 400 years, ensuring that the differences in ENSO characteristics due to significantly different coupling strengths are statistically significant.

Each of the 33 ensemble members of the perturbed parameter ensemble is characterised by a unique set of coupling parameter fields and noise characteristics. For each member, these terms are implemented in the ICM resulting in 33 unique versions. Sensitivity tests are also performed in which parameters are mutually exchanged between different ensemble members. This enables us to study the influence of the terms separately, test if the effects add linearly.

\subsection{SST-equation}

A linear local SST anomaly equation is used to parameterise SST variability. It describes the SST response to thermocline anomalies $Z_{20}^{\prime}$, the SST response to wind variability $\tau_{x}^{\prime}$ and damping on SST anomalies $T^{\prime}$. These processes have been separated by fitting the parameters in the equation:

$$
\begin{aligned}
\frac{d T^{\prime}}{d t}(x, y, t)= & \alpha(x, y) Z_{20}^{\prime}(x, y, t-\delta)+ \\
& \beta(x, y) \tau_{x}^{\prime}(x, y, t)-\gamma(x, y) T^{\prime}(x, y, t),
\end{aligned}
$$

to each of the ensemble members using least squares. Here, $\alpha$ is the SST response to thermocline anomalies, $\beta$ is the direct SST response to local wind variability and $\gamma$ is a damping term, including a latent heatflux and cloud feedbacks. As in van Oldenborgh et al. (2005), the finite upwelling time $\mathrm{d}$ is prescribed from observations (Zelle et al., 2005) and varies 
from less than one month east of $130 \mathrm{~W}$ to 5 months at the date line. This equation describes $60 \%-80 \%$ of the SST variability of the ensemble members in the equatorial Pacific region. Further away from the equator, Eq. (1) explains less than $40 \%$ of the SST variability in some areas. In those areas, values are tapered off to very small values for $\alpha$ and $\beta$ and to intermediate values for $\gamma$. A more detailed description of the SST-equation parameters is given in van Oldenborgh et al. (2005) and Philip and van Oldenborgh (2009).

Figure 2 shows the two-dimensional patterns for the STAM member. All terms in the SST-equation are important in the East Pacific near the coast of S. America. Away from this coastal region, the response of SST to thermocline anomalies $(\alpha)$ is largest in the central to eastern Pacific, the main region of SST anomalies in the model. In the West Pacific, both the response of SST to wind stress anomalies $(\beta)$ and the damping on SST $(\gamma)$ play an important role. While these patterns of response are, to leading order, similar to those found when fitting observation fields (Fig. 4 of Philip and van Oldenborgh, 2010), there are differences between the modelled and the observed patterns of parameters. The evaluation of ENSO in both flux-adjusted and non-flux adjusted versions of HadCM3 has been well documented (e.g. Spencer et al., 2007) and hence we do not perform extensive further analysis here. We simply note that HadCM3 is competitive with the performance of other models.

For the members of both the ATM- and OCN-ensembles, the spatial patterns of $\alpha, \beta$ and $\gamma$ are qualitatively similar to the STAM member but the magnitudes are different. For this reason it is appropriate to compare the ensemble members by averaging the values of the parameters in boxes distributed on longitude and centred on the equator (see Sect. 5).

\subsection{Statistical atmosphere model for zonal wind stress}

Another important term in the ENSO feedback loop is the response of the zonal wind stress to SST. This sensitivity can be fitted with a linear statistical atmosphere model:

$\tau_{x}^{\prime}(x, y, t)=\sum_{i=1}^{n} A_{i}(x, y) T_{i}^{\prime}(t)+\epsilon(x, y, t)$.

In this equation $\tau_{x}^{\prime}(x, y, t)$ describes the domain-wide zonal wind stress anomaly and $T_{i}^{\prime}(t)$ are SST anomalies averaged over separate regions $i=1,2, \ldots, n$ centred on the equator. The patterns $A_{i}(x, y)$ are the domain-wide wind stress patterns corresponding to these SST anomalies. The term $\epsilon(x, y, t)$ denotes the stochastic forcing by random wind stress variations (described in more detail in the next section).

Based on testing a number of different configurations, the responses of zonal wind stress to SST anomalies is best resolved in three boxes in the Pacific Ocean. Using more and smaller boxes gives rise to excessive noise in the response patterns and instabilities in the ICM. The effects of temperature anomalies in the three boxes on wind stress are thus
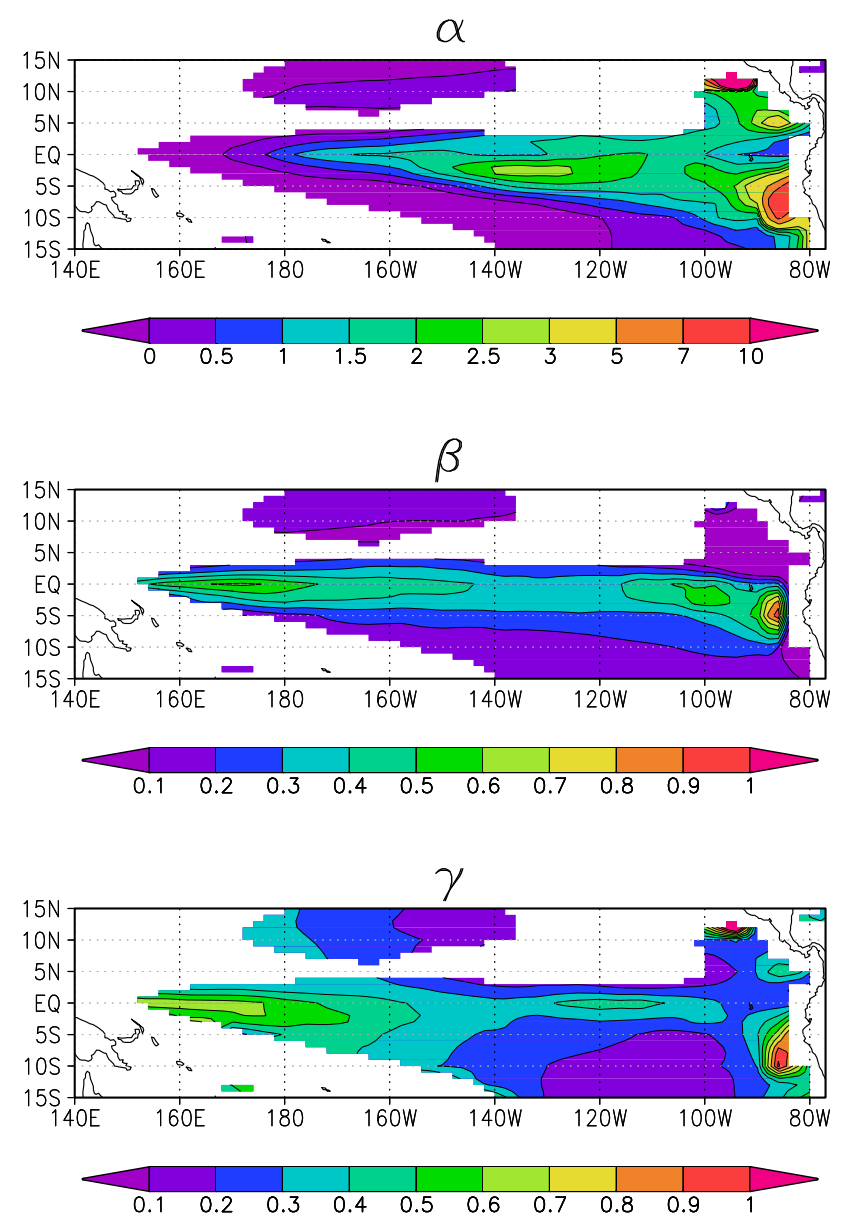

Fig. 2. Response of SST to thermocline anomalies, $\alpha$ $\left[0.1 \mathrm{Km}^{-1}\right.$ month $\left.^{-1}\right]$ (top) and to wind stress anomalies, $\beta$ $\left[100 \mathrm{~K} \mathrm{~Pa}^{-1}\right.$ month $\left.^{-1}\right]$ (middle) and the damping time on SST, $\gamma$ $\left[\right.$ month $^{-1}$ ] (bottom) for the STAM member. Only areas in which the SST-equation (Eq. 1) describes more than $40 \%$ of the SST variability are shaded. Note the nonlinear colour scale in the response of SST to thermocline anomalies.

investigated separately. For the linear statistical atmosphere model, this is mathematically equivalent to dividing the regression coefficient of wind stress on SST-index by the covariance of the SST-index: the three wind stress patterns then correspond to an SST anomaly in one of these three boxes only and not to anomalies in the other boxes. The patterns resemble somewhat Gill-type patterns (Gill, 1980), but differ in many details such as the relative strengths of the equatorial poles and the off-equatorial structure (see also Figs. 3 and 4 of van Oldenborgh et al., 2005).

Figure 3 shows the zonal wind stress response patterns for the STAM member using the three highlighted SST boxes. The wind stress response is always convergent towards the positive SST anomalies. This is consistent with a heating anomaly on top of a backgound temperature gradient and background wind (Clarke, 1994). The wind response west of 

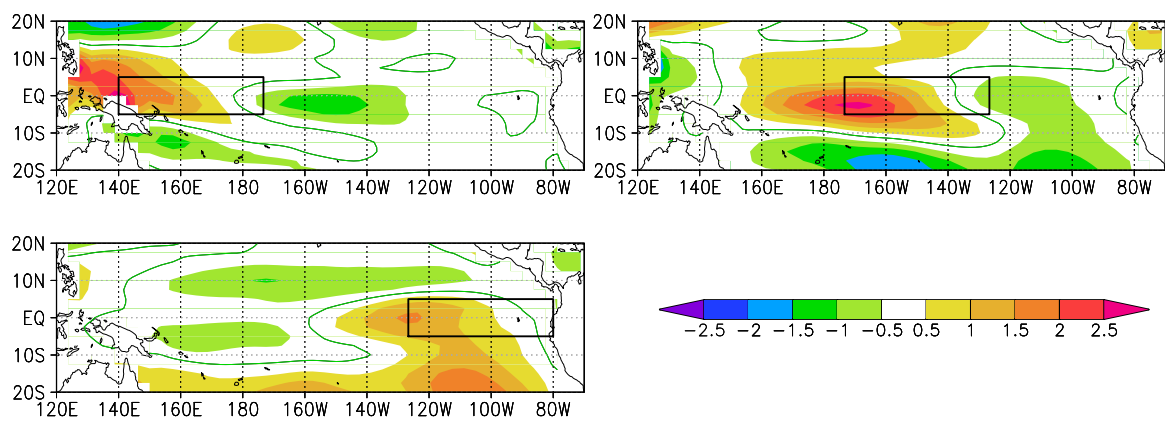

Fig. 3. The sensitivity of zonal wind stress anomalies to SST anomalies (term A in Eq. 2) of the STAM member $\left[10^{-3} \mathrm{Nm}^{-2} \mathrm{~K}^{-1}\right]$. The response is calculated using the three SST boxes highlighted on each figure panel. Orange-red colours correspond to eastward wind stress response to a positive SST anomaly in the indicated box, green-blue colours correspond to negative (westward) anomalies.

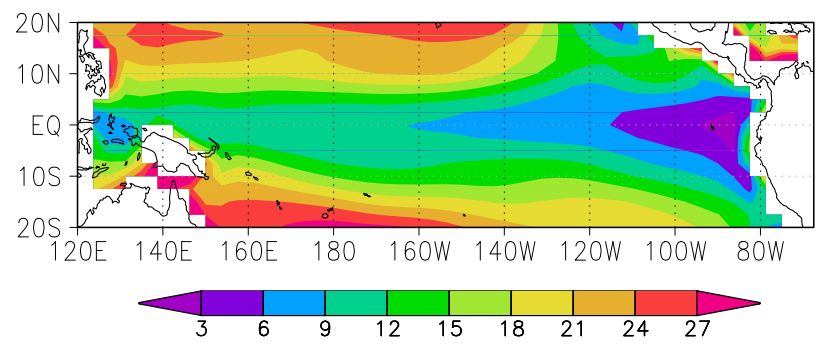

Fig. 4. Atmospheric noise standard deviation in $\left[10^{-3} \mathrm{Nm}^{-2}\right]$ of the STAM member.

the anomaly is stronger than the response east of the anomaly and the response to an SST anomaly in the east Pacific is weaker than that in the central West Pacific. The latter is due to the warmer background SST in the West Pacific relative to the East Pacific, which provides higher evaporation, more convection and consequently a stronger wind stress response to SST anomalies. The patterns and strength of the wind stress responses to the SST anomalies in the STAM member have a similar leading-order spatial structure to the observations (van Oldenborgh et al., 2005).

As in the case of the SST-equation parameters $\alpha, \beta$ and $\gamma$, the spatial patterns of $A_{i}$ for the perturbed members of ATMand OCN-ensemble are very similar to those for the STAM member shown in Fig. 3.

\subsection{Atmospheric noise properties}

In the ICM used in this study ENSO is stable and driven by external atmospheric noise derived from the GCM output. The external atmospheric noise $\epsilon(x, y, t)$ is defined as the residual of the total wind stress minus the wind stress calculated with Eq. (2): it is the component of the wind stress that is not directly correlated with SST anomalies. Philip and van Oldenborgh (2009) show that a physically consistent characterisation of this noise term is necessary. Therefore we describe the noise term with a two-dimensional pattern of noise amplitude and with a spatial and temporal autocorrelation.

Figure 4 shows the basin-wide amplitude of atmospheric noise for the STAM member. The noise amplitude is lowest in the eastern equatorial region where the background SST is lowest. The pattern resembles that calculated from observational data, but the amplitude is up to $40 \%$ lower near the equator compared to observations (Philip and van Oldenborgh, 2010).

In order to quantify the noise characteristics in each ensemble member, spatial and temporal correlation coefficients are estimated from 25 equally distributed locations between $30^{\circ} \mathrm{S}-30^{\circ} \mathrm{N}, 120^{\circ} \mathrm{E}-90^{\circ} \mathrm{W}$, divided in 5 locations zonally by 5 locations meridionally. This number of locations is enough to cover the whole basin with sufficient resolution. The distance at which the spatial correlation is less than 0.36 is calculated to be 24 degrees zonally and 4 degrees meridionally. A good approximation of the time-correlation coefficient at a lag of one month $a_{1}(x, y)$ is given by a function that varies linearly along the equator and exponentially along the meridionals as $a_{1}(x, y)=0.5\left(1+x / N_{x}\right) / \exp \left(\frac{1}{8}\left|y-2-\frac{1}{2} N_{y}\right|\right)$ with $x, y$ ranging from 1 to $N_{x}$ and 1 to $N_{y}$ respectively and $N_{x}=84, N_{y}=30$. Minimum values are set to 0.15 and maximum values, just north of the equator in the West Pacific, are cut off at 0.4 .

Again, the spatial patterns of atmospheric noise for the perturbed members of the ATM- and OCN-ensemble are very similar to those for the STAM member. However, as we see below, there are differences in the amplitudes of the patterns.

\subsection{Ocean component of the ICM}

The ocean component of the ICM uses a 1.5-layer ocean model with ocean wave dynamics described by the gravest baroclinic mode. The Kelvin wave speed is fitted to the ocean dynamical fields in the region $5^{\circ} \mathrm{S}-3^{\circ} \mathrm{N}, 150^{\circ} \mathrm{E}-110^{\circ} \mathrm{E}$, i.e. in the region where the correlation between the GCM thermocline amomalies and ICM thermocline anomalies is highest (Note that the Kelvin wave speed is a parameter of 
Table 1. ENSO characteristics in the perturbed parameter ensemble. The top row shows the characteristics from observations (obs) (Reynolds v2 SST). The error margin for the period is obtained from NCDC ERSST v3b data. In bold the STAM member. The amplitude $\langle\sigma\rangle$ is defined as the mean SST-standard deviation $\sigma\left[{ }^{\circ} \mathrm{C}\right.$ ] over $5^{\circ} \mathrm{S}-5^{\circ} \mathrm{N}, 160^{\circ} \mathrm{E}-100^{\circ} \mathrm{W}$. The mean period [years] is calculated from the timeseries of the average $\sigma$ over this box. For the ENSO pattern the longitude at which the $\sigma$ reaches a maximum $\sigma_{\max }$ in this box is given, together with a second maximum if this is approximately equally high. The term "broad" describes the describes the fact that the amplitude has no clear maximum but is zonally rather broad. Members 1-16 are part of the ATM-ensemble, members 17-32 describe the OCN-ensemble. Sampling errors in computing model values are of a similar order of magnitude as those computed for the observations.

\begin{tabular}{|c|c|c|c|c|c|c|c|c|c|}
\hline ATM & $\langle\sigma\rangle$ & period & $\begin{array}{l}\sigma_{\max } \\
\operatorname{lon}_{1}\end{array}$ & $\operatorname{lon}_{2}$ & OCN & $\langle\sigma\rangle$ & period & $\begin{array}{l}\sigma_{\max } \\
\operatorname{lon}_{1}\end{array}$ & $\operatorname{lon}_{2}$ \\
\hline obs & $0.93 \pm 0.13$ & $3.4 \pm 0.3$ & 255 & & & & & & \\
\hline 0 & 0.86 & 4.3 & 180 & & & & & & \\
\hline 1 & 1.16 & 4.8 & 195 & 251 & 17 & 1.18 & 4.1 & 224 & \\
\hline 2 & 1.04 & 4.2 & 191 & & 18 & 0.88 & 5.0 & 194 & \\
\hline 3 & 0.89 & 4.5 & 188 & & 19 & 0.88 & 5.5 & 232 & \\
\hline 4 & 0.93 & 3.8 & 180 & 229 & 20 & 0.99 & 4.1 & 194 & 244 \\
\hline 5 & 1.14 & 3.5 & 240 & & 21 & 0.86 & 4.2 & 198 & \\
\hline 6 & 0.74 & 3.6 & 180 & 248 & 22 & 0.92 & 4.3 & 194 & \\
\hline 7 & 0.57 & 4.2 & 154 & & 23 & 1.13 & 4.9 & 232 & \\
\hline 8 & 0.67 & 4.1 & 184 & 248 & 24 & 0.88 & 4.3 & 202 & \\
\hline 9 & 0.67 & 4.1 & 169 & & 25 & 1.00 & 4.1 & 232 & broad \\
\hline 10 & 0.83 & 3.9 & 176 & & 26 & 0.99 & 4.7 & 188 & \\
\hline 11 & 0.60 & 4.1 & 158 & & 27 & 1.22 & 4.8 & 218 & \\
\hline 12 & 0.86 & 4.4 & 191 & & 28 & 1.07 & 4.8 & 210 & \\
\hline 13 & 0.56 & 3.7 & 180 & 251 & 29 & 1.06 & 4.2 & 202 & 226 \\
\hline 14 & 0.79 & 4.0 & 183 & & 30 & 0.92 & 3.8 & 232 & \\
\hline 15 & 0.61 & 3.8 & 244 & & 31 & 1.01 & 4.7 & 210 & \\
\hline 16 & 0.80 & 4.2 & 180 & 244 & 32 & 0.99 & 4.0 & 210 & \\
\hline
\end{tabular}

the ICM. It is not fitted directly to oceanic Kelvin waves observed in the model simulations as these are affected by the coarse spatial resolution of the model. Rather it is a compact way of representing the behaviour of the 20 degree isotherm.). In this region, the thermocline is the principal driver of SST anomalies in comparison to the wind stress response to SST and in comparison to the damping. The SSTequation explains a large fraction of the variance $(>0.4)$ (see also Fig. 2).

The value for the Kelvin wave speed that results in the best-fit ocean dynamics is determined from a forced version of the ICM. In this version the SST-equation parameters in the ICM are fitted to all ensemble members separately. The forcing is represented by the two-dimensional zonal wind stress anomaly timeseries of the respective ensemble members. Different Kelvin wave speeds between $2.0 \mathrm{~m} \mathrm{~s}^{-2}$ and $2.6 \mathrm{~m} \mathrm{~s}^{-2}$ are tested for the highest average correlation between the ICM-thermocline depth and the thermocline depth of each ensemble member. For the STAM member the Kelvin wave speed that corresponds to the highest average correlation has a value of $2.4 \mathrm{~m} \mathrm{~s}^{-2}$. This is a realistic value compared to observations.

\section{Characteristics of modelled ENSO in the ensembles}

Firstly we examine a set of diagnostics of ENSO behaviour in the STAM and perturbed members of the ensemble. Commonly used diagnostics in the equatorial Pacific region relate to the ENSO amplitude, period and pattern. We define the ENSO pattern by the standard deviation of SST anomalies $\sigma$ and the amplitude is quantified by the average of $\sigma$ (denoted by $\langle\sigma\rangle$ ) over the region $5^{\circ} \mathrm{S}-5^{\circ} \mathrm{N}, 160^{\circ} \mathrm{E}-100^{\circ} \mathrm{W}$. (As most models, including HadCM3, tend to represent the cold tongue and the region with largest variability too far into the West Pacific, we choose a region that is larger than the common Niño3 or Niño3.4 boxes.) The mean period $\bar{T}$ is defined from the timeseries of the box-averaged SST anomalies over the region. The power spectrum of this timeseries is bandpass filtered between 1-10 year to filter out subseasonal and multi-decadal variability and then averaged by $\bar{T}=\exp \langle\log (1 / f)\rangle$, where the angular brackets denote the averaging with a weight proportional to the power at frequency $f$.

The ENSO characteristics of the perturbed parameter ensemble are listed in Table 1. These characteristics are in reasonable agreement with ENSO characteristics obtained from Reynolds SST observations (Reynolds et al., 2002). The amplitude of the STAM member $\left(0.86^{\circ} \mathrm{C}\right)$ is only slightly 
lower than that of observations $\left(0.93{ }^{\circ} \mathrm{C}\right)$. The mean period of 4.3 years is somewhat longer than that of observations (3.8 years), although estimating the period of such a complex oscillation can be significantly affected by sampling noise. In common with other GCMs, the maximum variability is too far to the west, although the displacement in this fluxadjusted HadCM3 is not as extreme as in, for example, the non-flux-adjusted version of HadCM3. ENSO characteristics of the perturbed parameter ensemble vary around the ENSO characteristics of the STAM member.

The mean climate in the ensemble can be described by the main actors in the ENSO phenomenon: SST, wind stress and thermocline depth. Additionally we calculate the mean mixed layer depth (MLD), as we need this later when we describe SST-equation parameters. To compare the mean climate of the ensemble members with the STAM member we defined a set of indices, all between $5^{\circ} \mathrm{S}-5^{\circ} \mathrm{N}$. The mean SST in the eastern Pacific $T_{\text {east }}$ is calculated between $127^{\circ} \mathrm{W}-85^{\circ} \mathrm{W}$ (3rd box in Eq. 2). An SST gradient $\Delta T$ is defined as the difference in SST between $127^{\circ} \mathrm{W}-$ $85^{\circ} \mathrm{W}$ and $140^{\circ} \mathrm{E}-172^{\circ} \mathrm{W}$ (3rd box minus 1 st box in Eq. 2). The mean wind stress and MLD are calculated for $140^{\circ} \mathrm{E}-$ $150^{\circ} \mathrm{W}\left(\tau_{x, \text { west }}\right)$ and $150^{\circ} \mathrm{W}-85^{\circ} \mathrm{W}\left(\tau_{x, \text { east }}\right)$, and the mean thermocline depth is defined for $180^{\circ}-150^{\circ} \mathrm{W}\left(H_{\text {central }}\right)$ and $130^{\circ} \mathrm{W}-85^{\circ} \mathrm{W}\left(H_{\text {east }}\right)$.

In general the differences in mean climate state between each perturbed member of the ensemble and the STAM are much smaller than the differences between the 19 structurally-different CMIP3 models examined in van Oldenborgh et al. (2005). Some variations around the STAM member are evident: a difference in $T_{\text {east }}$ with the STAM member ranging from $-0.5^{\circ} \mathrm{C}$ to $0.4^{\circ} \mathrm{C}$, an $\mathrm{SST}$ gradient that is at most $3.2 \mathrm{~K}$ larger than in the STAM member and a spread in thermocline depth between $74 \mathrm{~m}$ and $98 \mathrm{~m}$. The variation of the mean wind stress and of thermocline depth are correlated with variations in $\Delta T$, especially in the East Pacific. This is understood in terms of the well known balance between the pressure gradient force and the wind stress. The gradient in SST sets up the mean wind stress and this in turn influences the east-west gradient in thermocline depth.

Despite the similarities between the mean climates of the ensemble members that are imposed by the use of fluxadjustments, there are some subtle differences between individual members, which may impact the ENSO variability. The strongest correlation between the mean climate and ENSO characteristics is a relatively large correlation of -0.83 between $T_{\text {east }}$ and the ENSO amplitude for the ATMensemble, potentially due to variations in cloud processes across the ensemble (Toniazzo et al., 2008; Lloyd et al., 2009). However, for the OCN-ensemble this correlation is only -0.39 . We next examine the coupling characteristics in the ensemble members and their dependence on these subtle variations in the mean.

\section{ENSO coupling strength in the ensemble}

\subsection{Description of the SST-equation parameters}

The two-dimensional responses of SST to wind stress anomalies and thermocline anomalies and the damping coefficients are fitted to all the ensemble members. As noted above, whereas such patterns vary considerably between structurally different non-flux adjusted coupled GCMs (van Oldenborgh et al., 2005), the patterns are relatively similar across the ATM-ensemble and OCN-ensemble. Therefore it is possible to define indices for the amplitudes of the patterns that are used to quantify the differences in responses and damping terms. We can then check the dependence of the fitted parameters on the mean state.

To compare mean values of the responses and damping terms we average two regions where these terms are most important (see also Fig. 2). For all East Pacific terms, the east region is $5^{\circ} \mathrm{S}-5^{\circ} \mathrm{N}, 150^{\circ} \mathrm{W}-85^{\circ} \mathrm{W}$. For the response to wind stress anomalies and damping, the western-most region is defined as $5^{\circ} \mathrm{S}-5^{\circ} \mathrm{N}, 140^{\circ} \mathrm{E}-150^{\circ} \mathrm{W}$. For the $\mathrm{SST}$ response to thermocline anomalies, the western-most region is defined as $5^{\circ} \mathrm{S}-5^{\circ} \mathrm{N}, 180^{\circ}-150^{\circ} \mathrm{W}$.

The values are listed in Table 2 and Fig. 5 shows a selection of the most important relations between these terms and the mean climate. From previous studies (e.g. Fedorov and Philander, 2001; Philip and van Oldenborgh, 2006) we might expect that $\alpha$ (the response of SST to thermocline anomalies) will depend on the mean thermocline depth. Figure 5a shows that there is no such relation in the ATM-ensemble in either the east or the west. However, there is a relation between mean SST ( $\left.T_{\text {east }}\right)$ and $\alpha$ on both sides of the basin in the ATM ensemble (Fig. 5b). Taking into account the fact that there is no high correlation between mean thermocline depth and $T_{\text {east }}$, this relation must be explained by involving the vertical temperature gradient. When the mean SST is higher and the thermocline is equally deep the vertical temperature gradient is larger. This results in a stronger influence of thermocline anomalies on SST. In the OCN-ensemble we find a correlation of -0.76 between $\alpha$ and the mean thermocline depth in the East Pacific. The difference in this correlation between the ATM- and OCN-ensembles can be explained by the fact that $\alpha$ depends on both the vertical gradient and the thermocline depth. The standard deviation in mean SST is $0.25^{\circ} \mathrm{C}$ across the ATM-ensemble members and $0.16^{\circ} \mathrm{C}$ across the OCN-ensemble members. The standard deviation in mean thermocline depth is $2.9 \mathrm{~m}$ in the ATM-ensemble and $7.4 \mathrm{~m}$ in the OCN-ensemble. Perturbing ocean-model parameters apparently leads to more differences in mean thermocline depth than perturbing atmosphere-model parameters (potentially because of the use of flux adjustments). We find the highest correlations between $\alpha$ and mean SST in the ATMensemble and between $\alpha$ and mean thermocline depth in the OCN-ensemble. 
Table 2. Feedback parameters in $\%$ of change in the ATM-ensemble relative to the STAM member. The reference mean values of the parameters of the STAM member are also listed, with the values for the Kelvin wave speed $c_{o c}$ in $[\mathrm{m} / \mathrm{s}], \alpha$ in $\left[0.1 \mathrm{~K} \mathrm{~m}^{-1} \mathrm{month}^{-1}\right], \beta$ in $\left[100 \mathrm{~K} \mathrm{~Pa}^{-1}\right.$ month $\left.^{-1}\right], \gamma$ in $\left[\right.$ month $\left.^{-1}\right]$, the statistical atmosphere in $\left[10^{-3} \mathrm{Nm}^{-2} \mathrm{~K}^{-1}\right]$ and the atmospheric noise in $\left[10^{-3} \mathrm{Nm}^{-2}\right]$. Terms are defined in Eqs. (1) and (2).

\begin{tabular}{lrrrrrrrrrrrr}
\hline ATM & $c_{O C}$ & $\alpha_{\text {west }}$ & $\alpha_{\text {east }}$ & $\beta_{\text {west }}$ & $\beta_{\text {east }}$ & $\gamma_{\text {west }}$ & $\gamma_{\text {east }}$ & $A_{1}$ & $A_{2}$ & $A_{3}$ & $\epsilon_{\text {west }}$ & $\epsilon_{\text {east }}$ \\
\hline $\mathbf{0}$ & $\mathbf{2 . 4}$ & $\mathbf{0 . 0 9 1}$ & $\mathbf{0 . 1 7}$ & $\mathbf{0 . 3 0}$ & $\mathbf{0 . 3 2}$ & $\mathbf{0 . 4 3}$ & $\mathbf{0 . 2 9}$ & $\mathbf{1 3}$ & $\mathbf{1 5}$ & $\mathbf{1 0}$ & $\mathbf{1 1}$ & $\mathbf{6 . 7}$ \\
1 & -4 & -32 & -13 & -14 & 4 & -18 & -10 & -17 & 10 & 3 & 14 & 16 \\
2 & -4 & -28 & -9 & -8 & 9 & -4 & 2 & 5 & 7 & 12 & 17 & 14 \\
3 & -4 & -19 & -10 & -11 & -15 & -3 & -5 & 103 & 9 & 25 & 20 & 2 \\
4 & -4 & -4 & 6 & -3 & 0 & -8 & 6 & 17 & -5 & 15 & 3 & 0 \\
5 & -4 & -9 & 37 & 1 & 24 & 1 & 31 & -8 & -4 & 25 & 23 & 22 \\
6 & -4 & -4 & 5 & -12 & 4 & 2 & 18 & 101 & -5 & 11 & 17 & 8 \\
7 & -13 & 12 & 21 & 23 & 23 & -9 & 21 & 22 & -5 & -8 & -27 & -18 \\
8 & -8 & -5 & 5 & -27 & -8 & -3 & 11 & 60 & 20 & 10 & 20 & 10 \\
9 & -8 & -7 & 3 & 8 & 18 & -4 & 17 & -17 & 2 & -8 & -16 & -7 \\
10 & -4 & -6 & -3 & -9 & 2.8 & -10 & 1 & -6 & -2 & 7 & 5 & 1 \\
11 & -13 & 11 & 4 & 30 & 18 & -9 & 17 & 0 & 5 & -10 & -29 & -18 \\
12 & 4 & 49 & 4 & -5 & 34 & 0 & 0 & -2 & 11 & 1 & 11 & 4 \\
13 & -13 & 12 & 9 & -20 & 19 & -1 & 39 & 58 & 7 & 26 & 2 & -5 \\
14 & -4 & -16 & 12 & -12 & 12 & -1 & 25 & 39 & 15 & 20 & 18 & 12 \\
15 & -13 & 28 & -2 & -13 & -1 & 0 & 11 & 23 & 22 & 3 & -5 & -8 \\
16 & -4 & -11 & 0 & -16 & -15 & -1 & 3 & 98 & 4.3 & 10 & 27 & 10 \\
\hline
\end{tabular}

From Fig. 5c we find a correlation of -0.72 between the response of SST to wind stress anomalies $(\beta)$ and the mixed layer depth (MLD) in the OCN-ensemble. This is similar to what was found in the climate change scenario experiments (Philip and van Oldenborgh, 2006) where a shallower mixed layer depth results in a stronger response of SST to wind stress anomalies. A thinner mixed layer reacts more strongly to a wind anomaly than a thicker mixed layer. In the ATMensemble we see no significant correlation between the response of SST to wind stress anomalies and the mixed layer depth (recall that each member of the ATM ensemble uses the same ocean parameters). However, in the ATM-ensemble other parameters that influence $\beta$, e.g. processes which affect surface heat fluxes, are perturbed.

Finally, for higher mean SST we expected that clouds extend more to the east, resulting in stronger damping on SST. However, we do not find a relation between damping on SST and $T_{\text {east }}$ (see Fig. 5d), indicating that other terms influence the damping term. It should be noted that for the HadCM3 model, clouds and latent heat flux are equally important for the damping term (Philip and van Oldenborgh, 2006).

\subsection{Description of the statistical atmosphere model parameters}

From Eq. (2), the wind stress response to SST anomalies in three boxes along the equator is fitted for all the ensemble members. Again, the agreement between the spatial patterns is much higher than in the CMIP3 ensemble shown in van Oldenborgh et al. (2005) but the strength and meridional width do vary.
Differences between the models are described on the basis of four diagnostics. The first three are the amplitudes of the wind stress responses west of the three boxes to SST anomalies within the three boxes. The last one is the meridional width of the wind stress response west of the central box to an SST anomaly within this central box. The amplitudes of the wind stress responses are defined as averages over $\left(5^{\circ} \mathrm{S}-10^{\circ} \mathrm{N}, 130^{\circ} \mathrm{E}-170^{\circ} \mathrm{E}\right),\left(5^{\circ} \mathrm{S}-5^{\circ} \mathrm{N}, 160^{\circ} \mathrm{E}-150^{\circ} \mathrm{W}\right)$ and $\left(5^{\circ} \mathrm{S}-3^{\circ} \mathrm{N}, 150^{\circ} \mathrm{W}-100^{\circ} \mathrm{W}\right)$ for the three boxes respectively ( $A_{i}$ parameters fitted from Eq. 2 ). These values are listed in Table 2 . The meridional width of the wind stress response to an SST anomaly in the central box is defined by the meridional locations at which the domain-wide, zonally averaged wind stress response is zero (or, in some cases, reaches a minimum).

We might expect a warmer background temperature to provide higher evaporation and consequently a stronger wind stress response to SST anomalies ( $A_{i}$ in Eq. 2). The results of the ATM and OCN perturbed parameter ensemble show that there is no significant relation between background SST and wind stress response to SST anomalies at all, see Fig. 6a. Nevertheless, by perturbing parameters in the atmospherecomponent of the model it is possible to induce different levels of wind-stress response; a wider spread is evident in ATM when compared to the standard-atmosphere OCN ensemble. The perturbed parameters influence convective processes, for example, which may affect the sensitivity of evaporation to SST anomalies.

Kirtman (1997), Zelle et al. (2005) and Capotondi et al. (2006) showed that the period of ENSO depends on the 

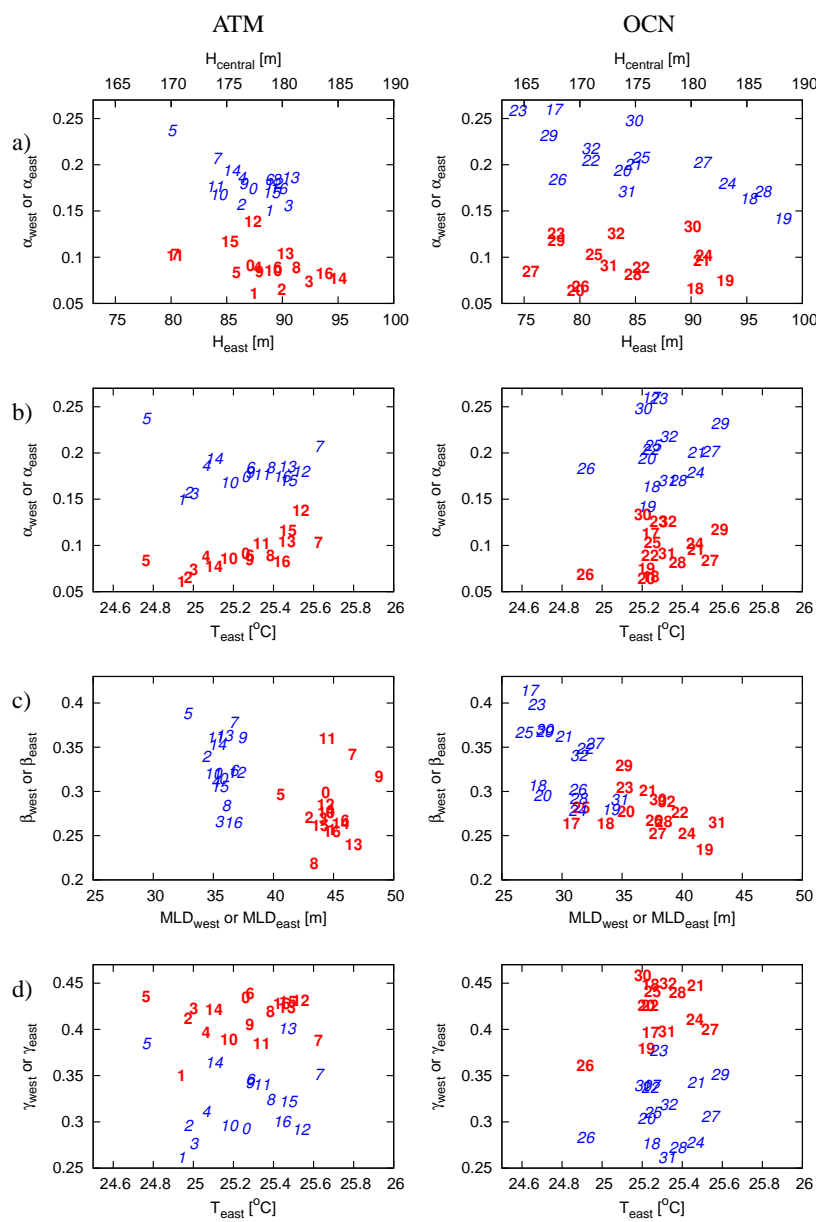

Fig. 5. Fitted SST-equation parameters as in Eq. (1) and Fig. 2 for all members, indicated by the numbers. Red are the values for the West Pacific, blue for the East Pacific. The left column shows the ATM ensemble, the right column shows the OCN ensemble. (a) Response of SST to thermocline anomalies, $\alpha[0.1 \mathrm{~K}$ $\mathrm{m}^{-1}$ month ${ }^{-1}$ ] versus mean thermocline depth. The labels for $H_{\text {central }}$ are plotted on top of the figures. (b) $\alpha$ versus mean East Pacific temperature. (c) SST response to wind stress anomalies, $\beta$ $\left[100 \mathrm{~K} \mathrm{~Pa}^{-1}\right.$ month $^{-1}$ ] versus mean mixed layer depth. (d) damping time on SST, $\gamma\left[\right.$ month $\left.^{-1}\right]$ versus mean East Pacific temperature.

meridional width of the wind stress response to SST. Figure $6 \mathrm{~b}$ shows that there indeed exists a weak relation within the perturbed parameter ensemble (correlation 0.45), although such a weak correlation can only partially explain the variations in ENSO period.

\subsection{Description of the atmospheric noise properties}

The wind stress noise, as defined in Sect. 3.4, has an amplitude and a spatial- and temporal-autocorrelation structure. The noise standard deviation pattern is similar for all the members of the ATM and OCN ensembles, with higher amplitudes in the East Pacific relative to the West Pacific (see
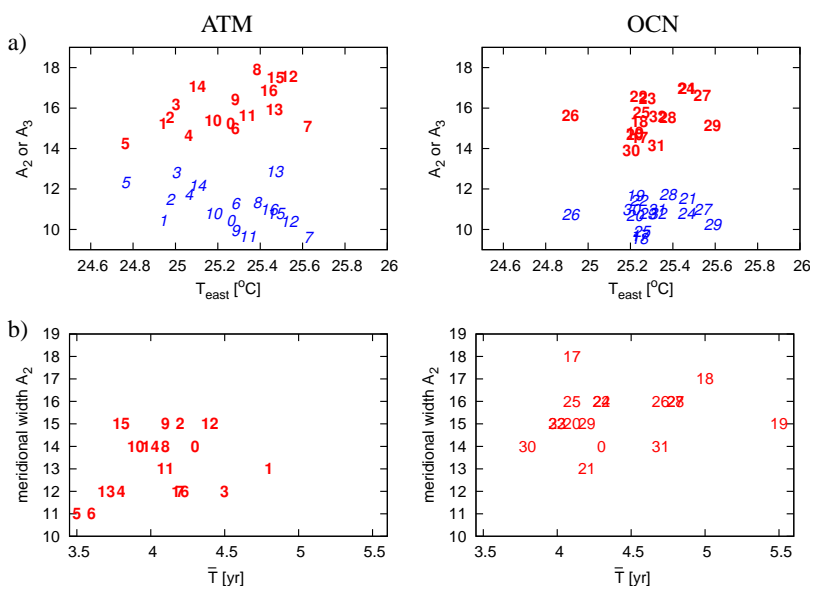

Fig. 6. (a) Statistical atmosphere box 2 and 3 in $\left[10^{-3} \mathrm{Nm}^{-2} \mathrm{~K}^{-1}\right]$ vs. SST for all members. Box 1 is not shown as this box shows no correlation with $T_{\text {east }}$ at all. Red are the western mean values, blue the eastern ones. The left column shows the ATM ensemble, the right column shows the OCN ensemble. (b) Meridional width of the response of wind stress to SST [degrees] in the central Pacific versus the mean period [years].

Fig. 4 for the STAM member). Variations are described below on the basis of average values over the regions $5^{\circ} \mathrm{S}-5^{\circ} \mathrm{N}$, $140^{\circ} \mathrm{E}-190^{\circ} \mathrm{E}$ and $5^{\circ} \mathrm{S}-5^{\circ} \mathrm{N}, 190^{\circ} \mathrm{W}-85^{\circ} \mathrm{E}$.

The standard deviation of the noise is the noisecharacteristic that varies most between the ensemble members. The spatial- and temporal-autocorrelation coefficients appeared to be relatively similar in each case. For the implementation of the noise field in the ICM (see later) it is thus possible to use one single set of autocorrelation coefficients to describe all ensemble members (see Philip and van Oldenborgh, 2009). We therefore focus on the analysis of the standard deviation of the noise. These values are listed in Table 2.

It can be seen from Table 2 and Fig. 7 that members 7 and 11 (ATM-ensemble) have very low noise levels. In both the West and East modest negative correlations, of -0.48 and -0.68 , are found between mean SST and noise amplitude in the ATM-ensemble respectively. In contrast, these correlations are positive in the $\mathrm{OCN}$-ensemble, with values of 0.55 and 0.45 respectively, although the spread in the noise is much smaller in OCN than in ATM. Perturbing atmospheremodel parameters results in a much wider variation of noise amplitudes, as might be expected, although the change of the sign of the correlation between noise amplitude and mean SST was not expected.

We expect that for higher noise levels the ENSO amplitude, $\langle\sigma\rangle$, becomes larger. Figure 7 confirms this positive correlation between noise and ENSO amplitude. For the ATM-ensemble in the East Pacific the correlation is 0.78. For the OCN-ensemble there is no strong correlation, as the variation in noise is relatively low. 


\subsection{Description of the gravest baroclinic mode}

A gravest mode equatorial Kelvin wave speed of $2.4 \mathrm{~m} / \mathrm{s}$ results in the best agreement between the ocean dynamics in the reference ICM and corresponding GCM STAM member (Sect. 3.5). For the other ensemble members most fitted values are somewhat lower (Table 2). A minimum value of $2.1 \mathrm{~m} / \mathrm{s}$ is fitted for members $7,11,13$ and 15 . As we see below, this ICM parameter has little influence on the behaviour of ENSO amplitude and pattern and can be held constant when using the ICM to reproduce the variability in SST amplitude and pattern of the GCM experiments.

\subsection{Summary of fitted ICM model parameters}

Parameter perturbations lead to variations in SST, wind and thermocline couplings, noise amplitude and damping on SST. In general, the variations in fitted ocean-parameters are larger in the OCN-ensemble and the variations in fitted atmosphere-parameters are larger in the ATM-ensemble. This is what we expect from the design of the perturbed parameter ensemble. In some cases these variations enhance each other in the influence on ENSO characteristics, e.g. a stronger noise amplitude and weaker damping tend to result in higher SST variability. In some specific members, both atmospheric noise and wind stress response to SST anomalies are weaker while SST responses to thermocline anomalies and wind stress anomalies are stronger and damping is weaker. This means that in these members the influence of the atmosphere is much smaller. Finally, we do not find simple correlations between the fitted components in the feedback loop and some of the main ENSO characteristics listed in Table 1. However, this might be the effect of compensating ENSO feedbacks, which masks the ultimate effect on the ENSO characteristics.

\section{Influence of feedback strengths on ENSO properties}

To investigate the effect of the variations of parameters across the ensembles on ENSO features and feedbacks, we run the ICM versions. Since the patterns of the components that are fitted in Sect. 5 are relatively similar, we can substitute the coupling strengths from one model version with those from another. This results in ICM versions that are fitted to a combination of, for example, the STAM member and one other perturbed physics member. This allows the isolation of specific features emerging from the simulations. We first investigate the most important ENSO characteristics of the ICM runs and compare them to the original GCM runs. Furthermore, we separate the contribution of each of the components to the ENSO properties into four categories. The first group includes the parameters of the SST-equation (Eq. 1), which include the responses of SST to wind and thermocline depth variability and damping. The second group describes the statistical atmosphere, with three boxes along the equator.
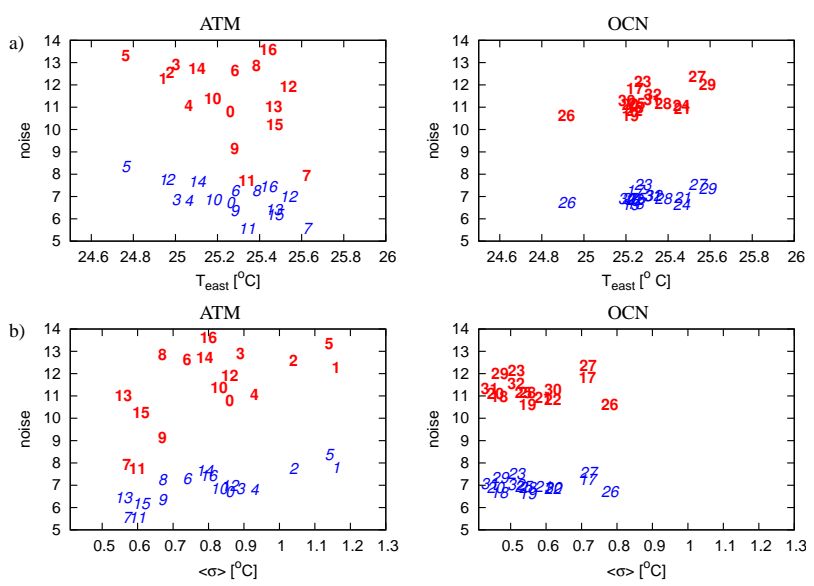

Fig. 7. (a) Atmospheric noise amplitude in $\left[10^{-3} \mathrm{Nm}^{-2}\right]$ vs. SST, $T_{\text {east }}$ and (b) vs. SST standard deviation (ENSO amplitude), $\langle\sigma\rangle$ for all members. Red are the western mean values, blue the eastern ones.

Thirdly, we study the influence of the atmospheric noise. Finally, the influence of the Kelvin wave speed is investigated. For clarity and readability we will mainly show results of the ATM-ensemble. However, we use both the ATM- and OCNensembles to draw conclusions.

\subsection{Verification of the ICM runs}

First, the ICM runs in which the whole feedback loop is fitted to one single ensemble member are investigated. For convenience we call this type of ICM experiment a "fullrun". Most ICM versions corresponding to ATM-ensemble members run well, except for the members 7, 9 and 11, in which the integration is numerically unstable. These are the models with very low SST standard deviation, a low noise amplitude and SST variability that is located too far in the West Pacific. It is possible to achieve numerical stability in these runs by adding an extra coupling term $\mu=0.82$ (members 7,11) or $\mu=0.95$ (member 9) between the ocean and atmosphere, such that in Eq. (2) $A_{i}(x, y)$ is replaced by $A_{i}^{\prime}(x, y)=\mu A_{i}(x, y)$. This allows us to show some qualitative results. However, as the coupling parameter changes the ICM runs and there is very little ENSO variability in both the HadCM3 and ICM runs we will not use these runs for a quantitative comparison. In the OCN-ensemble, the full-runs of members 17 and 26 need an extra coupling parameter of $\mu=0.90$ and $\mu=0.95$ respectively for the same reason.

Figure 8a shows the SST standard deviation patterns of the ICM full-runs and the original GCM ensemble members. We note that, as found in previous studies, the ICM does not simulate off-equatorial SST variability well as it is only a conceptual model of equatorial ENSO processes.

Close to the equator, the SST variability simulated with the ICM is slightly lower than the original GCM SST variability. 


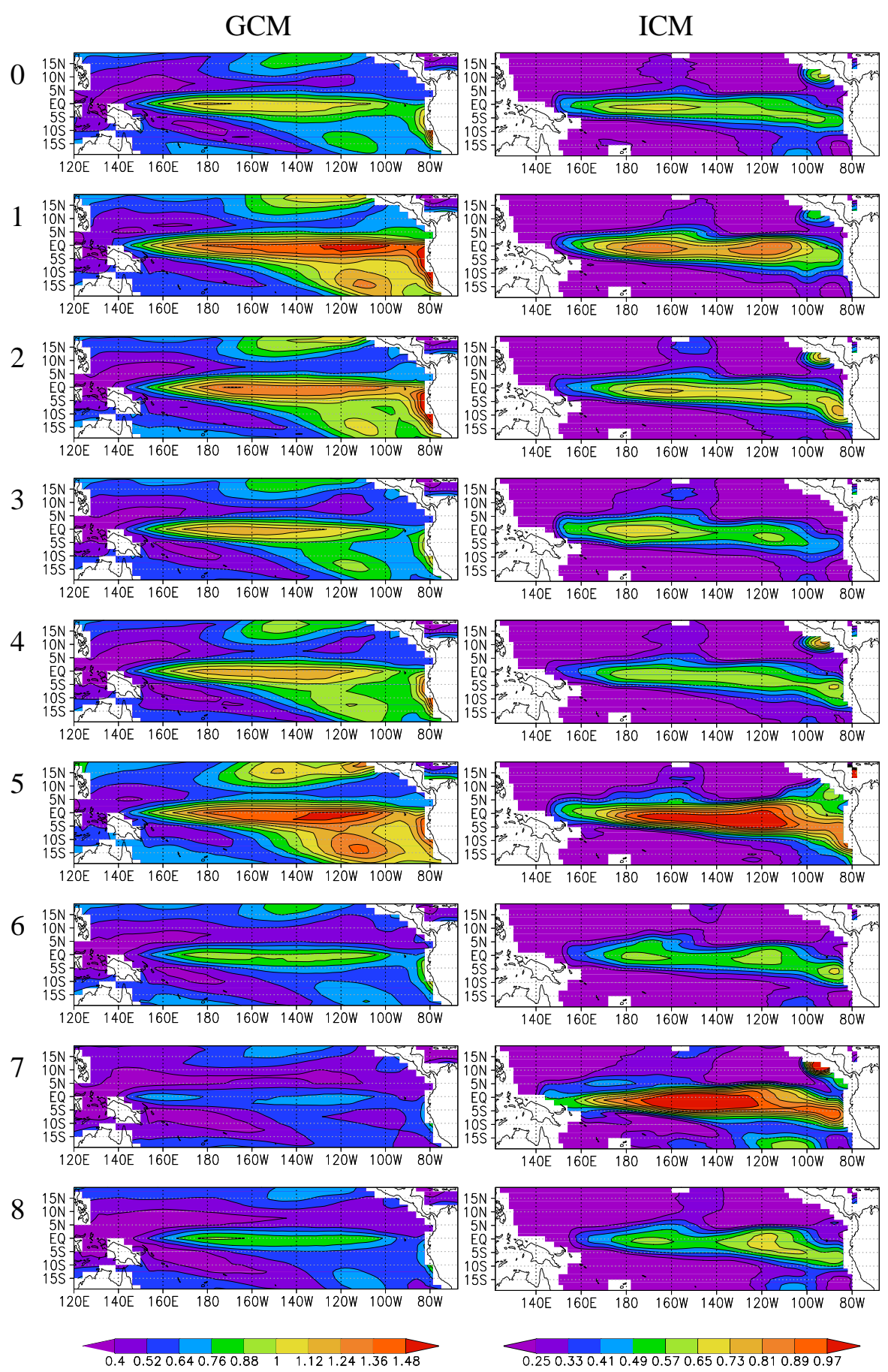

Fig. 8a. Pattern of SST standard deviation for GCM ensemble members (left) and corresponding full ICM runs (right): STAM-member and ATM-members 1-9. Note the factor of $2 / 3$ difference in scale.

Although not all SST standard deviation patterns of the ICM runs resemble the patterns calculated from GCM output, there are some similarities. For instance, in members STAM, 10,12 and 16 the maximum is located in the central to West Pacific, in member 5 the maximum is further to the East Pacific, and in member 6 there are two clear maxima in both the east and the west. For other members, the agreement between the GCM and ICM variability is not as good.

In order to quantify the resemblance we consider the maximum of SST standard deviation and the corresponding location (Fig. 9). With the exception of members 7, 9 and 11 (noted above), there is a clear relation between the locations 

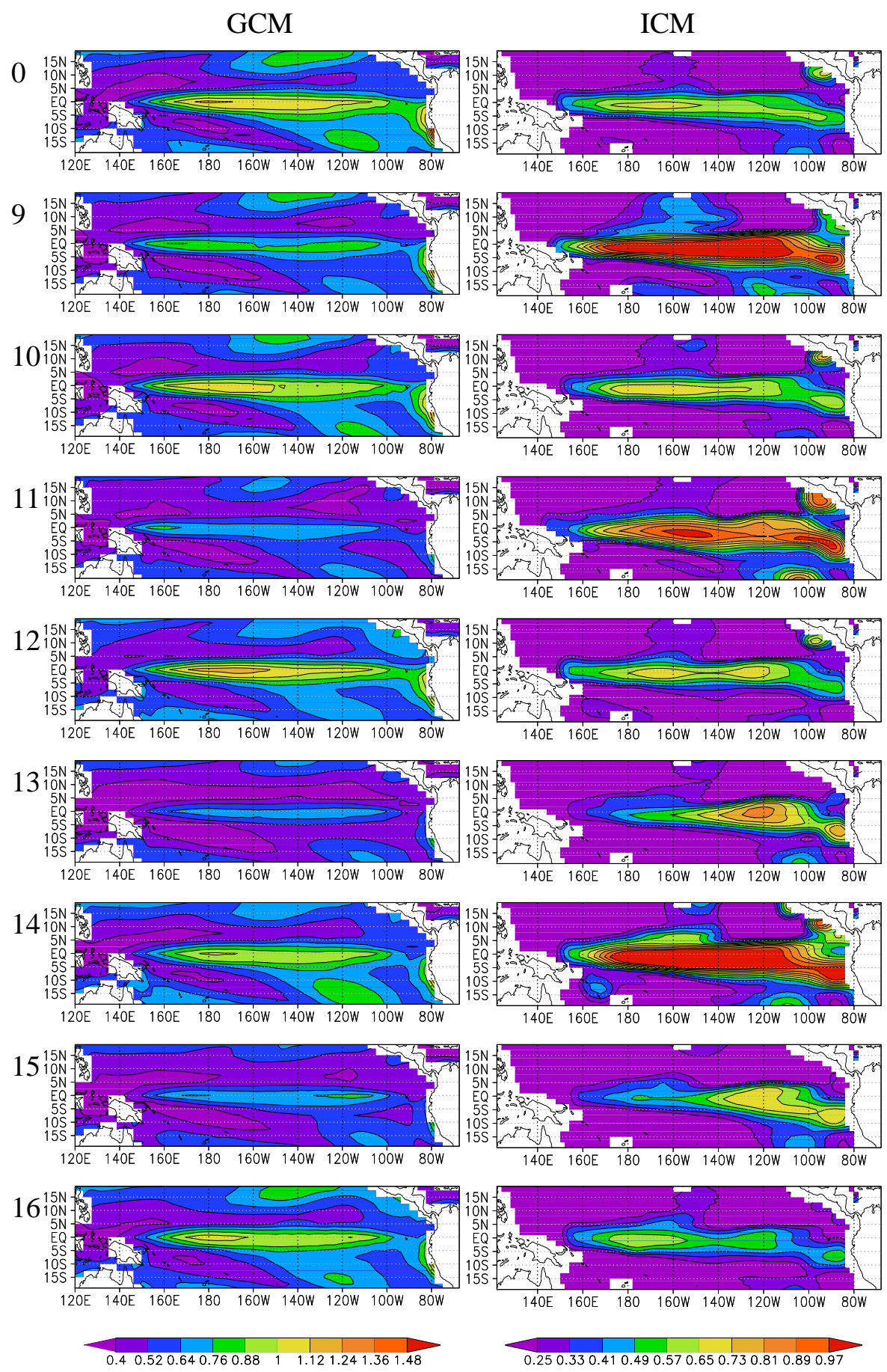

Fig. 8b. STAM-member and ATM-members 9-16.

of the maximum SST standard deviation of the ATM-fullruns. For the ATM- and OCN-full-runs together the correlation is 0.88 . Except for members 13, which has an unrealistic low SST variability, and 14, which is a clear outlier, the correlation between the maximum SST variability of the GCM runs and ICM runs is also positive, although the ICM ensemble displays systematically lower values than the GCMs and the data are not distributed along a 1:1 line. For member 13 the problem is similar to that of members 7, 9 and 11: the ICM is not able to capture the unrealistically low ENSO variability. The exceptionally high ENSO amplitude for the ICM run of member 14 is due to the statistical atmosphere (see next section). 

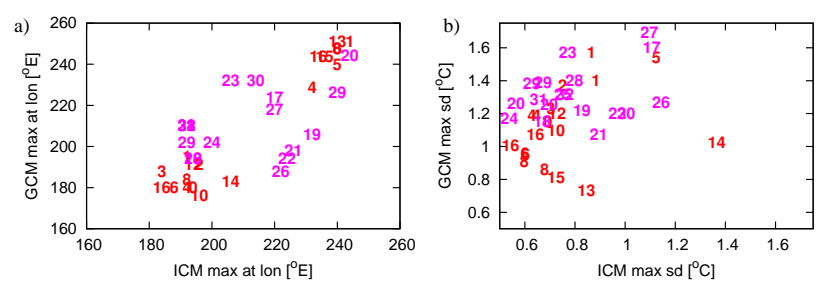

Fig. 9. (a) Location of maximum SST standard deviation in degrees E. If two locations exist in both GCM and ICM run, both are plotted. (b) Amplitude at the location of maximum SST standard deviation of GCM ensemble members vs. corresponding ICM runs. In red the ATM-full-runs and in pink the OCN-full-runs.

We note here that there is no correlation between GCM ENSO period and the corresponding fitted ICM ENSO period in these experiments (figure not shown). This reveals a weakness in the ICM approach which needs to be addressed in future research. The ICM ENSO period is principally determined by the phase speed of the gravest baroclinic Kelvin wave, which, as we note above, has little spread when computed from the GCM experiments. Another factor influencing the period is the meridional width of the wind stress response to SST variability. Nevertheless, there is a modest spread in ENSO period from the GCM ensemble experiments. The ENSO period has also been hard to reproduce in many other studies. We omit discussion of the period of ENSO in what follows.

Overall, the ICM runs capture the amplitude and spatial ENSO characteristics. This is sufficient to use them as a basis to better understand the influence of the parameter perturbations on ENSO by investigating the relative contribution of the different couplings on ENSO characteristics.

\subsection{Contribution of feedback strengths to ENSO}

Having established that ENSO properties are only weakly correlated with the mean state in this ensemble, we proceed to investigate the direct effects on ENSO of the various couplings defined in Fig. 1. Note again that in the CMIP3 ensemble, these two effects were inextricably intertwined. Due to the flux-corrected mean state of the perturbed physics ensemble there is the potential for greater separation of mean state errors and coupling processes. For instance, we can investigate the influence of the set of SST-equation parameters of the ATM-ensemble on ENSO by running the ICM with parameters fitted to the STAM member and then varying the parameters in one of the components in the feedback loop. In Fig. 10 we show SST standard deviation patterns $\sigma$ of a selection of six ensemble members that illustrates this investigation. For each member the upper three panels show the $\sigma$ of the ICM runs in which either the set of SST-equation parameters or the statistical atmosphere parameters or the atmospheric noise are changed. (For member 11 the extra cou- pling of $\mu$ is used in all runs.) With this method we disentangle the influence of the different components of the feedback loop on ENSO amplitude and pattern. The fourth panel shows again the $\sigma$ of the ICM full-run in which all components are fitted to one GCM ensemble member. The three intermediate panels are compared to the reference ICM and the full-run ICM.

Runs in which only the Kelvin wave speed is changed are not shown, as the change in $\sigma$ is mainly seen in small variations in the amplitude and period and not in the pattern, and the differences between the ICM versions are not large. Using a Kelvin wave speed of $2.3 \mathrm{~m} / \mathrm{s}$ instead of the standard value of $2.4 \mathrm{~m} / \mathrm{s}$ results in an amplitude that is only $0.03 \mathrm{~K}$ higher than the reference amplitude of $0.50 \mathrm{~K}$.

Figure 10 shows that the different SST variability results from a combination of changes. In most cases the different components of the feedback loop add almost linearly. The SST variability, $\langle\sigma\rangle$, of GCM ensemble member 2 is larger than in the STAM member, while the pattern is relatively similar. In the ICM this is reproduced correctly. A lower $\langle\sigma\rangle$ would be expected based on the values of the statisticalatmosphere parameters, but this is counteracted by the higher $\langle\sigma\rangle$ resulting from higher atmospheric noise.

In member 5 the GCM SST variability is located further to the east than in the standard member (which is more like in reality). From the ICM runs we learn that this is caused mainly by the SST-equation parameters. The two distinct maxima seen in SST variability in member 6 are mainly caused by the values of the statistical-atmosphere parameters.

Considering the pattern of variability in GCM member 11, we see that the responses described by the SST-equation are responsible for the SST variability being located much too far in the West Pacific in the GCM (see also Fig. 8b). In this ensemble member the damping of SST anomalies $\gamma$ is extraordinarily low. Further investigation shows that this is mainly due to a very low latent heat flux sensitivity to SST variations.

The SST variability in member 12 suggests that this member is almost similar to the STAM member. However, this is a combination of much higher SST variability from the responses described by the SST-equation, compensated by much lower SST variability caused by a weaker atmospheric response to SST anomalies. Finally, the lower SST variability in member 16 is the result of both changes in the ocean and the atmosphere, which is not entirely repaired by the higher noise level. It seems that there is some compensation between the different feedback loops such that the range of possible ENSO behaviour is reduced.

The behaviour of members 7, 9 and 11 is rather exceptional. Compared to the STAM member, these members have both weaker noise and weaker or similar wind stress response to SST anomalies. Moreover, the ocean parameters $\alpha$ and $\beta$ are larger and the damping is weaker in the West Pacific and stronger in the East Pacific. This results in much lower SST 

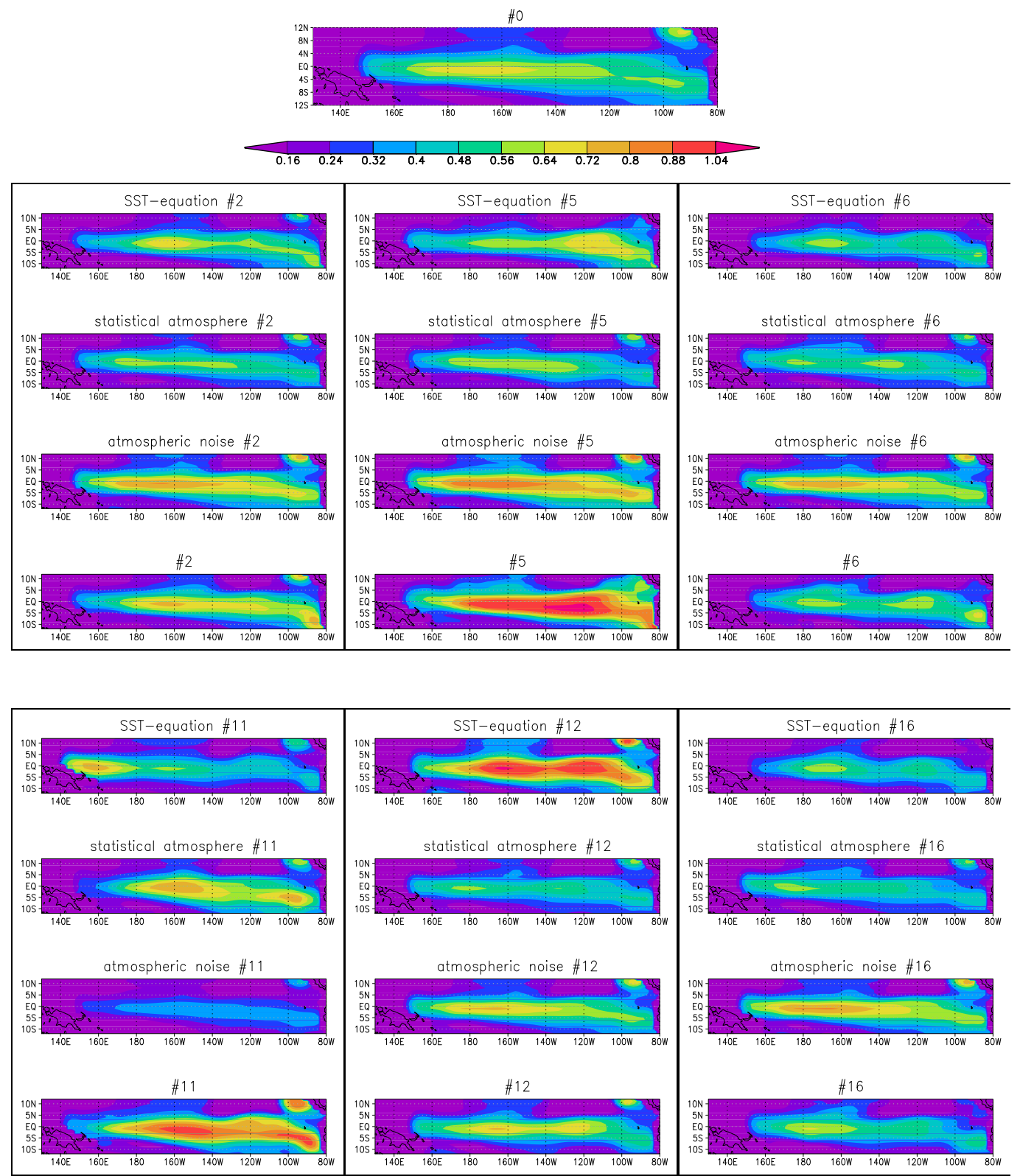

Fig. 10. SST standard deviation $\sigma[\mathrm{K}]$ for a selection of ICM fits to the GCM ensemble members but isolating the influence of different components of the ICM. Top: $\sigma$ for the STAM member 0 and colorbar. In each of the panels for members 2, 5, 6, 11, 12 and 16, from top to bottom we show $\sigma$ in an ICM run with only the SST-equation parameters from the perturbed member, but with the other ICM parameters held fixed at the standard values; $\sigma$ in an ICM run with statistical-atmosphere parameters from that member and all other ICM parameters held fixed at the standard values; $\sigma$ in an ICM run with atmospheric noise parameters from that member and all other ICM parameters held fixed at the standard values and $\sigma$ from the full-runs (reproducing the fields in Fig. 9). Note that 11 uses an extra coupling $\mu=0.82$. The influence of the Kelvin wave speed is not shown as it only results in a change in amplitude. See also text.

variability, with maximum SST variability far in the West Pacific. This SST variability is no longer directly related to El Niño. As our conceptual model is based on ENSO dynamics, we suspect that our approach is not valid for these three members.
The ICM full-runs of the GCM OCN-ensemble member 17 and 26 are numerically unstable. For member 17 , we can attribute this to the SST-equation parameters. Combining SST-equation parameters of the STAM member with all other parameters of member 17 results in a stable ICMversion. We could potentially add a nonlinear damping term 
that counterbalances the high responses of SST to thermocline and wind stress variability in the East Pacific in this member. In member $26, T_{\text {east }}$ is very low compared to the rest of the OCN-ensemble. We do not think that this is caused by the numerical instability of the ICM. Replacing an arbitrary part of the feedback loop of the full-run with member 26 by the parameters of the STAM-member results in a stable ICM.

Overall, we conclude that the SST-equation parameters and atmosphere response to SST anomalies affect both the ENSO amplitude and the pattern of variability. The noise amplitude (without significantly modifying the noise pattern) has a small influence on the ENSO amplitude.

We can quantify the influence of the different components in the feedback cycle on ENSO by studying different groups; in each group of ICM-runs only one set of coupling parameters is varied. Table 3 shows the influence of each group of coupling strengths in the ENSO feedback loop; the SSTequation parameters, the statistical atmosphere parameters, the atmospheric noise ampitude and the Kelvin wave speed. Besides the total influence of the whole SST equation, we also quantify the influence of the thermcline coupling $\alpha$, the direct wind coupling $\beta$ and the damping $\gamma$ in Eq. (1) separately. We ignore nonlinear interactions between the components of the feedback loop, but Fig. 10 and other analyses presented indicate that this is a reasonable assumption.

To determine the influence of the set of SST-equations on ENSO, we substitute the set of SST-equation parameters with the sets of parameters for all ICM boxes derived from the 16 ATM-ensemble members. We calculate the average amplitude of the SST variability, $\langle\sigma\rangle$ of these 16 ICM runs and subtract $\langle\sigma\rangle_{\text {STAM }}$, which gives us 16 numbers giving a distribution around the $\langle\sigma\rangle$ of the STAM-ICM. The width (standard deviation) of this distribution is a measure of the variation in ENSO amplitude accomplished by changing only one component in the feedback loop. This measure is given as a percentage of the width of the equivalent distribution of $\langle\sigma\rangle$ that is obtained when the full perturbations are used in the ICM ensemble. We perform a similar analysis in the OCNensemble. In principle the results depend strongly on the (subjective) choice of perturbed parameters in the ensemble. In practice, however, the results for the ATM and OCN ensembles are very well comparable, which implies that the results are quite insensitive to parameter choices. Results are summarised in Table 3. The influence of the components on the SST variability pattern is given qualitatively.

In the perturbed physics ensembles studied here, the changes in Kelvin wave speed do not influence ENSO amplitude and pattern very much. Surprisingly, neither does the noise amplitude, in spite of the good correlations found in the CMIP3 ensemble between noise amplitude and ENSO amplitude (Philip and van Oldenborgh, 2010). The spread of properties of the atmospheric response and SST equation explain most of the spread of the ensembles.

The influence of the three SST-equation parameters separately is larger than the combined variation, which means that
Table 3. Influence of different components in the feedback loop on ENSO amplitude and pattern in the ICM when varying the parameters in different ICM components based on the values fitted from the GCM experiments. Values are calculated as the spread in the amplitude, expressed as a percentage change from the STAM-member accomplished by varying one of the components in the feedback loop separately. Absolute values for the amplitude $\langle\sigma\rangle$ for the STAM member are given in the top row. For the pattern, we qualitatively assess the influence of the components as ranged from very little influence (0) to largest influence (++). Absolute values for the amplitude $\langle\sigma\rangle$ in the east Pacific (Table 1) for the STAM member are given in the top row. For the SST-equation parameters a the total contribution is listed as well as the influence of the three parameters separately.

\begin{tabular}{llcc}
\hline & parameter & $\langle\sigma\rangle$ & pattern \\
& reference & $\mathbf{0 . 5 0}$ & \\
\hline \multirow{2}{*}{ ATM } & SST-equation & 20 & ++ \\
& statistical atmosphere & 28 & + \\
& $\alpha$ & 12 & + \\
& $\beta$ & 24 & ++ \\
& $\gamma$ & 44 & ++ \\
& atmospheric noise & 10 & 0 \\
OCN & Kelvin wave speed & 8 & 0 \\
& SST-equation & 19 & ++ \\
& statistical atmosphere & 19 & + \\
& $\alpha$ & 23 & + \\
& $\beta$ & 36 & ++ \\
& $\gamma$ & 36 & +++ \\
& atmospheric noise & 4 & 0 \\
& Kelvin wave speed & 4 & 0 \\
\hline
\end{tabular}

variations in SST-equation parameters counteract each other (in agreement with Lloyd et al., 2009). The largest variability in ENSO amplitude $\langle\sigma\rangle$ is obtained by the variations in the damping $\gamma$. In the OCN ensemble this is equal to the influence of variations in the direct wind coupling $\beta$. In both ensembles the variability in the response of SST to thermocline anomalies $(\alpha)$ has a smaller influence.

Comparing the influence of parameter perturbations on ENSO amplitude between the ATM and OCN ensemble we conclude that the influence of the variability in SST response to thermocline variations $\alpha$ is largest in the OCN ensemble. This is what we expect from perturbing ocean parameters. Secondly, the zonal wind feedback $\beta$ depends strongly on the ocean mixed layer depth, resulting in a larger influence this parameter $\beta$ on ENSO in the OCN ensemble. Thirdly, atmospheric parameter perturbations lead to a larger influence of variations in the statistical atmosphere on SST in the ATM ensemble than in the OCN ensemble.

To investigate the processes behind the large variability of the damping term $\gamma$ we separated out the latent and shortwave (cloud) feedback components. ATM ensembles members with strong SW radiation feedback have reduced SST 
variability in the West Pacific. The strength of this feedback varies by more than a factor of two in the ATM ensemble and seems an important factor in determining the westward extend of SST variability. As expected, the OCN ensemble has a much smaller spread of feedback strengths. The models with high SST variability in the West Pacific have a stronger contribution from latent heat flux damping. Further investigation of the relative roles of SW, LW and other surface flux feedbacks in different geographical areas and their possible cancellation would be an interesting area of future research.

We conclude that the ocean and atmosphere parameters affect both the ENSO amplitude and the pattern of variability in this GCM ensemble. For the amplitude, the influence of the SST-equation parameters is approximately equivalent to the influence of the parameters that control the statistical atmosphere. However, the influence of damping of SST anomalies $\gamma$ and the response of SST to wind stress variability $\beta$ on SST are larger than the combined parameter settings. For the spatial pattern, the influence of the SST-equation parameters is greater. The role of the atmospheric noise amplitude and ocean dynamics on the spread of ENSO amplitude and spatial structure is relatively smaller.

\section{Conclusions}

We have quantified the role of various components in the ENSO feedback loop on the amplitude and pattern of ENSO variability. In most multi-model studies these couplings affect both the mean state and the ENSO couplings, making it difficult to separate the influences. Here, we used two fluxcorrected perturbed physics ensembles to negate the effects of mean state changes to first order. This allows us to study the effects of the parameter changes on the ENSO cycle directly.

The two ensembles are variants of the HadCM3 climate model with perturbations to either the parameters of the atmosphere model (ATM-ensemble) or perturbations to ocean parameters (OCN-ensemble). Both ocean-atmosphere couplings and atmospheric noise terms are directly impacted by the parameter perturbations, the noise terms more so in the case of the ATM-ensemble. The spread in ENSO characteristics does not show one-to-one relations with the spread in the mean climate variables, as might be expected from imposing flux adjustments in the ensemble runs, which tend to produce mean climates which are, to leading order, similar in each member. Rather the leading-order impact of the parameter perturbations is to affect ENSO coupling strengths directly, independently from the mean climate. (However, for non-linear processes such as the threshold triggering of convection, there may be more subtle interactions between the mean state and the physical processes that are perturbed. Such non-linearities are not invistgated here.)
An Intermediate Complexity Model (ICM) in which the main ENSO feedbacks are fitted to one GCM ensemble member or to a combination of ensemble members is employed to illuminate the GCM behaviour. Based on a number of diagnostics we can say that the ICM successfully reproduces the behaviour of 28 out of 33 ensemble members. The influence of four different components of ENSO is studied one by one. These components include SST-equation parameters, covering the response of SST on thermocline anomalies, wind stress anomalies and damping on SST, the response of wind stress on SST anomalies, a gravest baroclinic Kelvin wave speed in the ocean and the amplitude of atmospheric noise. The SST-equation parameters influence the pattern and amplitude of SST variability most, followed by the response of wind stress to SST anomalies. The influence of the SST-equation parameters separately is larger than the influence of the combination of parameters, which means that they counteract each other. The influence of the amplitude of atmospheric noise and the Kelvin wave speed on the ENSO pattern is much smaller. However, both factors do contribute to the ENSO amplitude. We observe that coupling strengths between the ocean and atmosphere tend to counteract each other, thereby reducing the potential range of variability in ENSO characteristics that might have been realised without this compensating feedback.

We can speculate on the mechanisms leading to the difference in coupling parameters in the GCM ensemble. Atmospheric parameter perturbations influence the ocean as well. The variations in $\alpha$, the SST response to thermocline variations, are due to changes in the the shallow ocean stratification that is influenced by atmospheric model parameters. These also affect the SST response to zonal wind stress anomalies, the mean wind stress $\beta$ through the mean wind stress and the mixed layer depth. Finally, cloud and atmospheric boundary layer parametrisations strongly affect the damping of SST anomalies through latent heat flux and cloud formation, especially in the western Pacific but also in the East (although the effects are harder to disentangle because of the large SST variability in this region).

The oceanic parameter perturbations influence the atmosphere via the SST, which is affected by changes in ocean surface currents, ocean mixed layer depth and temperature. Via this pathway oceanic parameter settings impact atmospheric coupling parameters in the ENSO feedback cycle, such as the response of wind stress to SST anomalies, atmospheric noise characteristics and cloud feedbacks. Due to the indirect pathway, the variation in this response is smaller in the OCN ensemble than in the ATM ensemble.

An incredibly useful potential for studies such as this is to improve climate models by linking the attributes of the model climate, in this case the attributes of ENSO, to the individual parameters and parameterisation schemes. Unfortunately, the relatively small number of ensemble members in comparison to the relatively large number of parameters sampled here means that it is not possible to separate the impact 
of the individual parameters in this way. With several hundred ensemble members, it is possible to "emulate" aspects of the mean climate of versions of HadCM3 that are coupled to a simple slab ocean (Rougier et al., 2009). This will be the subject of future research.

In this study perturbations to atmospheric and oceanic physics cause a spread in ENSO characteristics that can be related to the spread in ocean-atmosphere coupling strengths. This is somewhat different from previous work on multimodel studies and climate change scenarios. In multi-model studies both mean climates and ocean-atmosphere coupling strengths differ (Guilyardi, 2006; van Oldenborgh et al., 2005; Merryfield, 2006), which makes it difficult to discuss the effect of physical parameters separately (although recently Lloyd et al. (2009) have made some progress). In climate change scenario studies model parameters other than those related to climate change are not varied (Philip and van Oldenborgh, 2006).

The main conclusion is that, in the ensemble studied here, independent of the mean state, the largest uncertainties in the modelled amplitude and pattern of ENSO are in the sensitivity of SST to local wind in the central Pacific and damping of SST anomalies. The wind stress response to SST anomalies also plays a major role. The influence of the sensitivity of SST to thermocline depth in the eastern Pacific on ENSO is slightly smaller. Variations in modelled weather noise properties and Kelvin wave speed do not contribute much to the model uncertainty of ENSO properties.

Acknowledgements. This research was supported by the Research Council for Earth and Life Sciences (ALW) of the Netherlands Organisation for Scientific Research (NWO). Mat Collins was supported by the joint DECC, Defra and MoD Integrated Climate Programme - DECC/Defra (GA01101), MoD (CBC/2B/0417_Annex C5).

Edited by: D. Stevens

\section{References}

AchutaRao, K. and Sperber, K. R.: Simulation of the El Niõ Southern Oscillation: Results from the Coupled Model Intercomparison Project, Clim. Dynam., 19, 191-209, 2002.

AchutaRao, K. and Sperber, K. R.: ENSO simulation in coupled ocean-atmosphere models: are the current models better?, Clim. Dynam., 27, 1-15, 2006.

Annan, J. D., Hargreaves, J. C., Ohgaito, R., Abe-Ouchi, A., and Emori, S.: Efficiently constraining climate sensitivity with ensembles of paleoclimate simulations, Scientific on-line letters on the atmosphere, 1, 181-184, 2005.

Burgers, G., Balmaseda, M. A., Vossepoel, F. C., van Oldenborgh, G. J., and van Leeuwen, P. J.: Balanced Ocean-Data Assimilation near the Equator, J. Phys. Oceanogr., 32, 2509-2529, 2002.

Burgers, G. J. H. and van Oldenborgh, G. J.: On the impact of local feedbacks in the Central Pacific on the ENSO cycle, J. Climate, 16, 2396-2407, 2003.
Capotondi, A., Wittenberg, A., and Masina, S.: Spatial and temporal structure of tropical Pacific interannual variability in 20th century coupled simulations, Ocean Model., 15, 274-298, 2006.

Clarke, A. J.: Why Are Surface Equatorial ENSO Winds Anomalously Westerly under Anomalous Large-Scale Convection?, J. Climate, 7, 1623-1627, 1994.

Collins, M.: Ensembles and probabilities: a new era in the prediction of climate change, Philosophical Transactions of the Royal Society A, 365(1857), 1957-1970, 2007.

Collins, M., Tett, S. F. B., and Cooper, C.: The internal climate variability of HadCM3, a version of the Hadley Centre coupled model without flux adjustments, Clim. Dynam., 17, 61-81, 2001.

Collins, M., Booth, B., Harris, G., Murphy, J., Sexton, D., and Webb, M.: Towards Quantifying Uncertainty in Transient Climate Change, Clim. Dynam., 27(2-3), 127-147, 2006.

Dewitte, B., Cibot, C., Périgaud, C., An, S. I., and Terray, L.: Interaction between Near-Annual and ENSO Modes in a CGCM Simulation: Role of the Equatorial Background Mean State, J. Climate, 20, 1035-1052, 2007.

Neelin, J. D. and Dijkstra, H. A.: Ocean-atmosphere interation and the tropical climatology. Part 1: The dangers of flux correction, J. Climate, 8, 1325-1342, 1995

Fedorov, A. V. and Philander, S. G.: A stability analysis of tropical ocean-atmosphere interactions: bridging measurements and theory for El Niño, J. Climate, 14, 3086-3101, 2001.

Gill, A. E.: Some simple solutions for heat induced tropical circulation, Q. J. Roy. Meteor. Soc., 106, 447-462, 1980.

Gill, A. E.: Atmosphere-Ocean Dynamics, Academic Press, Orlando, 662 pp., 1982.

Gordon, C., Cooper, C., Senior, C. A., Banks, H., Gregory, J. M., Johns, T. C., Mitchell, J. F. B., and Ingram, W. R.: The simulation of SST, sea ice extents and ocean heat transport in a version of the Hadley Centre coupled model without flux adjustments, Clim. Dynam., 16, 147-168, 2000.

Guilyardi, E.: El Niño - mean state - seasonal cycle interactions in a multi-model ensemble, Clim. Dynam., 26, 329-348, 2006.

Guilyardi, E., Wittenberg, A., Fedorov, A., Collins, M., Wang, C., Capotondi, A., van Oldenborgh, G. J., and Stockdale, T.: Understanding El Niño in Ocean-Atmosphere General Circulation Models: progress and challenges, B. Am. Meteorol. Soc., 90, 325-340, 2009.

Harris, G., Sexton, D., Booth, B., Collins, M., Murphy, J., and Webb, M.: Frequency Distributions of Transient Regional Climate Change from Perturbed Physics Ensembles of General Circulation Model Simulations, Clim. Dynam., 27, 357-375, 2006.

Kirtman, B.: Oceanic Rossby wave dynamics and the ENSO period in a coupled model, J. Climate, 10, 1690-1704, 1997.

Knutti, R., Meehl, G. A., Allen, M. R., and Stainforth, D. A.: Constraining climate sensitivity from the seasonal cycle in surface temperature, J. Climate, 19, 4224-4233, 2006.

Lin, J. L.: The Double-ITCZ Problem in IPCC AR4 Coupled GCMs: Ocean-Atmosphere Feedback Analysis, J. Climate, 20, 4497-4525, 2007.

Lloyd, J., Guilyardi, E., Weller, H., and Slingo, J.: The Role of Atmosphere Feedbacks During ENSO in the CMIP3 Models, Atmos. Sci. Lett., 10(3), 170-176, 2009.

Merryfield, W. J.: Changes in ENSO under $\mathrm{CO}_{2}$ doubling in the IPCC AR4 coupled climate models, J. Climate, 19, 4009-4027, 2006. 
Moore, B., Gates, W., L. J., M., and Underdal, A.: Advancing our understanding, in: Climate Change 2001: The Scientific Basis. Contribution of Working Group I to the Third Assessment Report of the Intergovernmental Panel on Climate Change, edited by: Houghton, J. T., Ding, Y., Griggs, D. J., Noguer, M., van der Linden, P. J., Dai. X., Maskell, K., and Johnson, C. A., Cambridge University Press, 2001.

Murphy, J. M., Sexton, D. M. H., Barnett, D. N., Jones, G. S., Webb, M. J., Collins, M., and Stainforth, D. A.: Quantification of modelling uncertainties in a large ensemble of climate change simulations, Nature, 430, 768-772, 2004.

Niehörster, F., Spangehl, T., Fast, I., and Cubasch, U.: Quantification of model uncertainties: Parameter sensitivities of the coupled model ECHO-G with middle atmosphere, Vol. 8, EGU06A-08526, 2006.

Philip, S. and van Oldenborgh, G. J.: Shifts in ENSO coupling processes under global warming, Geophys. Res. Lett., 33, L11704, doi:10.1029/2006GL026196, 2006.

Philip, S. and van Oldenborgh, G. J.: Significant atmospheric nonlinearities in the ENSO cycle, J. Climate, 22, 4014-4028, 2009.

Philip, S. Y. and van Oldenborgh, G. J.: Atmospheric properties and ENSO: models versus observations, Clim. Dynam., doi:10.1007/s00382-009-0579-7, in press, 2010.

Piani, C., Frame D. J., Stainforth, D. A., and Allen, M. R.: Constraints on climate change from a multi-thousand member ensemble of simulations, Geophys. Res. Lett., 32, L23825, doi:10.1029/2005GL024452, 2005.

Reynolds, R. W., Rayner, N. A., Smith, T. M., Stokes, D. C., and Wang, W.: An Improved In Situ and Satellite SST Analysis for Climate, J. Climate, 15, 1609-1625, 2002.

Rougier, J., Sexton, D., Murphy, J., and Stainforth, D.: Analysing the climate sensitivity of the HadSM3 climate model using ensembles from different but related experiments, J. Climate, 22(13), 3540-3557, doi:10.1175/2008JCLI2533.1, 2009.

Sanderson, B., Knutti, R., Aina, T., Christensen, C., Faull, N., Frame, D., Ingram, W., Piani, C., Stainforth, D. A. and Stone, D., and Allen, M.: Constraints on Model Response to Greenhouse Gas Forcing and the Role of Subgrid-Scale Processes, J. Climate, 21, 2384-2400, 2008.

Sanderson, B. M. and Piani, C.: Towards constraining climate sensitivity by linear analysis of feedback patterns in thousands of perturbed-physics gcm simulations, Clim. Dynam., 30, 175-190, 2007.
Schneider von Deimling, T., Held, H., Ganopolski, A., and Rahmstorf, S.: Climate sensitivity estimated from ensemble simulations of glacial climates, Clim. Dynam., 27, 149-163, 2006.

Spencer, H., R. Sutton and J. M. Slingo: El Nio in a Coupled Climate Model: Sensitivity to Changes in Mean State Induced by Heat Flux and Wind Stress Corrections, J. Climate, 20, 2273 2298, 2007.

Stainforth, D. A., Aina, T., Christensen, C., Collins, M., Faull, N., Frame, D. J., Kettleborough, J. A., Knight, S., Martin, A., Murphy, J. M., Piani, C., Sexton, D., Smith, L. A., Spicer, R. A., Thorpe, A. J., and Allen, M. R.: Uncertainty in predictions of the climate response to rising levels of greenhouse gases, Nature, 433, 403-406, 2005.

Timmermann, A., Okumura, Y., An, S.-I., Clement, A., Dong, B., Guilyardi, E., Hu, A., Jungclaus, J., Krebs, U., Renold, M., Stocker, T. F., Stouffer, R. J., Sutton, R., Xie, S.-P., and Yin, J.: The influence of a weakening of the Atlantic meridional overturning circulation on ENSO, J. Climate, 20, 4899-4919, 2007.

Toniazzo, T.: Properties of the El Niño Southern Oscillation in different equilibrium climates with the HadCM3 model, J. Climate, 19, 4854-4876, 2006.

Toniazzo, T., Collins, M., and Brown, J.: The variation of ENSO characteristics associated with atmospheric parameter perturbations in a coupled model, Clim. Dynam., 30, 643-656, 2008.

van Oldenborgh, G. J., Philip, S. Y., and Collins, M: El Niño in a changing climate: a multi-model study, Ocean Sci., 1, 81-95, 2005 , http://www.ocean-sci.net/1/81/2005/.

Webb, M. J., Senior, C. A., Sexton, D. M. H., Ingram, W. J., Williams, K. D., Ringer, M. A., McAvaney, B. J., Colman, R., Soden, B. J., Gudgel, R., Knutson, T., Emori, S., Ogura, T., Tsushima, Y., Andronova, N., Li, B., Musat, I., Bony, S., and Taylor, K. E.: On the contribution of local feedback mechanisms to the range of climate sensitivity in two GCM ensembles, Clim. Dynam., 27, 17-18, 2006.

Zelle, H., Van Oldenborgh, G. J., Burgers, G., and Dijkstra, H. A.: El Niño and Greenhouse Warming: Results from Ensemble Simulations with the NCAR CCSM, J. Climate, 18, 4683-4669, 2005. 